\title{
NONAUTONOMOUS LINEAR-QUADRATIC DISSIPATIVE CONTROL PROCESSES WITHOUT UNIFORM NULL CONTROLLABILITY
}

\author{
RUSSELL JOHNSON, SYLVIA NOVO, CARMEN NÚÑEZ, AND RAFAEL OBAYA
}

\begin{abstract}
In this paper the dissipativity of a family of linear-quadratic control processes is studied. The application of the Pontryagin Maximum Principle to this problem gives rise to a family of linear Hamiltonian systems for which the existence of an exponential dichotomy is assumed, but no condition of controllability is imposed. As a consequence, some of the systems of this family could be abnormal. Sufficient conditions for the dissipativity of the processes are provided assuming the existence of global positive solutions of the Riccati equation induced by the family of linear Hamiltonian systems or by a convenient disconjugate perturbation of it.
\end{abstract}

\section{INTRODUCTION}

In this paper the dissipativity of a time-dependent linear control system

$$
\mathbf{x}^{\prime}=A(t) \mathbf{x}+B(t) \mathbf{u}
$$

where $\mathbf{x} \in \mathbb{R}^{n}$ and $\mathbf{u} \in \mathbb{R}^{m}$, together with the quadratic supply form

$$
\mathcal{Q}(t, \mathbf{x}, \mathbf{u})=\frac{1}{2}(\langle\mathbf{x}, G(t) \mathbf{x}\rangle+2\langle\mathbf{x}, g(t) \mathbf{u}\rangle+\langle\mathbf{u}, R(t) \mathbf{u}\rangle),
$$

is studied. The functions $A, B, G, g$ and $R$ are assumed to be bounded and uniformly continuous functions on $\mathbb{R}$, with values in the sets of real matrices of the appropriate dimensions. In addition, $G$ and $R$ are symmetric, and $R \geq \delta I_{m}$ for a fixed $\delta>0$.

The concept of dissipative dynamical system was introduced by Willems in [34, $32]$ in terms of an inequality involving the so-called storage function and the supply form. Generally speaking, a dissipative system is characterized by the property that at any time the amount of energy which it has dispersed in the environment (which can be measured in terms of the variation of the storage function) can not exceed the amount of energy that has been supplied to it (measured by the integration of the supply form). This notion has interest both from a theoretical and a practical point of view. It has been intensively investigated in systems theory and other related areas (see Fabbri et al. [5], Hill [8], Hill-Moylan [9], Polushin [24] and Savkin-Petersen [31]).

The linear control system (1.1) with quadratic supply form (1.2) can be included in a standard way in a family of control systems

$$
\mathbf{x}^{\prime}=A(\omega \cdot t) \mathbf{x}+B(\omega \cdot t) \mathbf{u},
$$

Key words and phrases. Nonautonomous linear Hamiltonian systems, Linear-quadratic control system, dissipativity, abnormal system, rotation number, proper focal point.

Partly supported by MIUR (Italy), by MEC (Spain) under project MTM2012-30860, and by JCyL (Spain) under project VA118A12-1. 
with the corresponding family of quadratic forms

$$
\mathcal{Q}_{\omega}(t, \mathbf{x}, \mathbf{u})=\frac{1}{2}(\langle\mathbf{x}, G(\omega \cdot t) \mathbf{x}\rangle+2\langle\mathbf{x}, g(\omega \cdot t) \mathbf{u}\rangle+\langle\mathbf{u}, R(\omega \cdot t) \mathbf{u}\rangle),
$$

for $\omega \in \Omega$, where $\Omega$ is a compact metric space with a continuous flow $\sigma$, and $A, G: \Omega \rightarrow \mathbb{M}_{n \times n}(\mathbb{R}), B, g: \Omega \rightarrow \mathbb{M}_{n \times m}(\mathbb{R})$ and $R: \Omega \rightarrow \mathbb{M}_{m \times m}(\mathbb{R})$ are continuous matrix-valued functions, with $G$ and $R$ symmetric and $R>0$. The results regarding the dissipativity of the initial system (1.1) can be derived from the results concerning the family (1.3). The advantage of this collective formulation is illustrated in Fabbri et al. [6,5] and Johnson-Núñez [15].

The quadratic form (1.4) gives rise to a quadratic functional by integrating:

$$
\widetilde{\mathcal{I}}_{\omega}(\mathbf{x}, \mathbf{u})=\int_{0}^{T} \mathcal{Q}_{\omega}(t, \mathbf{x}(t), \mathbf{u}(t)) d t
$$

where $0<T \leq \infty$ and $\mathbf{x}(t)$ is the solution of (1.3) determined by a generic control function $\mathbf{u}$ and a fixed initial vector $\mathbf{x}_{0} \in \mathbb{R}^{n}$ (thus $\mathbf{x}(0)=\mathbf{x}_{0}$; in the end $\mathbf{x}_{0}$ is allowed to vary). The standard LQ (linear-quadratic) control problem consists in minimizing $\widetilde{\mathcal{I}}_{\omega}(\mathbf{x}, \mathbf{u})$ subject to (1.3). It was noted by Yakubovich et al. [36] that there is a close relation between the infinite-horizon $(T=\infty)$ LQ control problem and the problem of the existence of a storage function for (1.1) and (1.2). They assumed that the coefficients $A, B, G, g, R$ are $T$-periodic functions of time; their results were extended to the case of general time-varying coefficients in [5].

The application of the Pontryagin Maximum Principle to the study of the LQ control problem defined by (1.3) and $\widetilde{I}_{\omega}$ gives rise to the family of nonautonomous linear Hamiltonian systems

$$
\mathbf{z}^{\prime}=H(\omega \cdot t) \mathbf{z}
$$

for $\omega \in \Omega$, where $\mathbf{z}=\left[\begin{array}{l}\mathbf{x} \\ \mathbf{y}\end{array}\right]$ for $\mathbf{x}, \mathbf{y} \in \mathbb{R}^{n}$ and

$$
H(\omega)=\left[\begin{array}{cc}
A(\omega)-B(\omega) R^{-1}(\omega) g^{T}(\omega) & B(\omega) R^{-1}(\omega) B^{T}(\omega) \\
G(\omega)-g(\omega) R^{-1}(\omega) g^{T}(\omega) & -A^{T}(\omega)+g(\omega) R^{-1}(\omega) B^{T}(\omega)
\end{array}\right],
$$

which induces skew-product flows on the linear bundle $\Omega \times \mathbb{R}^{2 n}$ and on the Lagrangian bundle $\Omega \times \mathcal{L}_{\mathbb{R}}$, as explained in Section 2 .

Fabbri et al. [5] and Johnson-Núnez [15] study the dissipativity of (1.3) with supply rate (1.4) in terms of the dynamical structure of this induced Lagrangian flow. They assume that the family (1.5) admits an exponential dichotomy (Frequency Condition, as defined in [6]) and that the family of control processes (1.3) is uniformly null controllable. Under these conditions, the family (1.5) is uniformly weakly disconjugate and the Lagrange planes associated to the exponential dichotomy do not lie on the Maslov cycle (Nonoscillation Conditions for (1.5) and its time reversed family, as defined in [6]). Equivalently, they admit representations of the form $l^{-}(\omega) \equiv\left[\begin{array}{c}I_{n} \\ M^{-}(\omega)\end{array}\right]$ and $l^{+}(\omega) \equiv\left[\begin{array}{c}I_{n} \\ M^{+}(\omega)\end{array}\right]$ for each $\omega \in \Omega$. Here, $l^{ \pm}(\omega)$ represent the vector spaces of the solutions of (1.5) which are bounded as $t \rightarrow \pm \infty$, respectively. They prove that " $M^{-}(\omega) \geq 0$ for each $\omega \in \Omega$ " is a necessary and sufficient condition for the dissipativity of the family of control processes (1.3) with supply form (1.4). In addition, the control processes are strictly dissipative if and only if $M^{-}(\omega)>0$ for every $\omega \in \Omega$. The study of the periodic case was carried out by Yakubovich et al. in [36]. The papers ([5], [15]) treat the general time-varying case. 
In the present paper we assume again the existence of an exponential dichotomy for the family (1.5) but the assumption of uniform null controllability is removed. We extend some of the previous criteria based on the positivity of the $M$-function and we also obtain some characterizations of the dissipativity in terms of the corresponding $M$-functions of an appropriate perturbation of the family (1.5). Thus, we provide a quadratic storage function in a new and more complex scenario. Since, in the absence of uniform null controllability, some of the associated linear Hamiltonian systems of the family must be abnormal, a section of the paper is devoted to the study of this kind of system.

We next explain the structure and main results of the paper. In Section 2 we recall the main notions of topological dynamics and ergodic theory which we will need. Section 3 is devoted to the study of the structure of a family of linear Hamiltonian systems containing abnormal systems. We prove that the index of abnormality is a semicontinuous invariant function admitting a residual invariant set of continuity points where it is locally constant, and it takes its minimum there. The maximum value is attained on appropriate minimal subsets of $\Omega$ where it is constant and greater or equal than 1 . In addition, if the family admits an exponential dichotomy, one of the Lagrange planes associated to the exponential dichotomy lies in the Maslov cycle. Hence, the Nonoscillation Conditions for (1.5) and its timereversed system do not hold simultaneaously in this scenario. The rotation number for linear Hamiltonian systems is a robust parameter with dynamical significance, we refer the reader to Johnson [10], Johnson and Nerurkar [11] and Novo et al. [23] for its study and applications. In this paper we obtain a representation of the rotation number which is useful for the abnormal case, in terms of the multiplicity of proper focal points of a conjoined basis.

In Section 4 we define the dissipativity of a control system (1.1) with supply rate (1.2) in the terms given by Willems [34], and provide a nonautonomous version of the construction of the available storage in this context. When the family (1.3) is uniformly null controllable, the dissipativity of the system corresponding to $\omega$ with supply rate (1.4), is equivalent to the statement that $\int_{t_{1}}^{t_{2}} \mathcal{Q}_{\omega}(s, \mathbf{x}(s), \mathbf{u}(s)) d s \geq 0$ for each pair $t_{1}<t_{2}$, control $\mathbf{u}:\left[t_{1}, t_{2}\right] \rightarrow \mathbb{R}^{m}$ and solution $\mathbf{x}(t)$ of (1.3) satisfying $\mathbf{x}\left(t_{1}\right)=0$. Thus, we can understand the integral of the supply form as a power function. Also under the assumption of uniform null controllability, we show that if the dissipativity holds for a point $\omega_{0}$ with dense orbit, it holds for each $\omega \in \Omega$. It is not hard to observe that both results are false when the controllability condition is omitted and, in consequence, dissipative and non dissipative control processes can coexist in the family (1.3). Some criteria, aimed at dealing with this new situation, and based on the existence of a global solution of the Riccati equation induced by the Lagrangian flow, are also given in this section.

This last section also contains criteria for dissipativity when the family of linear Hamiltonian systems (1.5) has exponential dichotomy, in two cases: first, when the Lagrange plane $l^{-}(\omega)$ admits a representation of the form $\left[\begin{array}{c}I_{n} \\ M^{-}(\omega)\end{array}\right]$, with $M^{-}(\omega) \geq 0$ for each $\omega \in \Omega$, without further assumptions on the family of Lagrange planes $\left\{l^{+}(\omega) \mid \omega \in \Omega\right\}$ (i.e., the Nonoscillation Condition for (1.5) can fail); and second, without any additional hypothesis on $l^{ \pm}$, in the sense that both Lagrange planes could globally or partially lie in the vertical Maslov cycle, but assuming the existence of an appropriate disconjugate $\varepsilon$-perturbation of (1.5) for 
which $M_{\varepsilon}^{-}$globally exists and is positive semidefinite. Finally, some examples show the applicability of the results.

\section{Preliminaries}

This section begins by recalling some basic concepts and properties on topological dynamics and ergodic theory, which are discussed in Ellis [3] and Cornfeld et al. [2]. Let $\Omega$ be a complete metric space. A (real and continuous) global flow on $\Omega$ is a continuous map $\sigma: \mathbb{R} \times \Omega \rightarrow \Omega,(t, \omega) \mapsto \sigma(t, \omega)$ such that $\sigma_{0}=\operatorname{Id}$ and $\sigma_{s+t}=$ $\sigma_{t} \circ \sigma_{s}$ for each $s, t \in \mathbb{R}$, where $\sigma_{t}(\omega)=\sigma(t, \omega)$. The flow is local if the map $\sigma$ is defined, continuous, and satisfies the previous properties on an open subset of $\mathbb{R} \times \Omega$ containing $\{0\} \times \Omega$.

Let $(\Omega, \sigma)$ be a global flow. The $\sigma$-orbit of a point $\omega \in \Omega$ is the set $\left\{\sigma_{t}(\omega) \mid t \in\right.$ $\mathbb{R}\}$. Restricting the time to $t \geq 0$ or $t \leq 0$ leads to the definition of forward or backward $\sigma$-semiorbit. A subset $\Omega_{1} \subset \Omega$ is $\sigma$-invariant (resp. positively $\sigma$-invariant or negatively $\sigma$-invariant) if $\sigma_{t}\left(\Omega_{1}\right)=\Omega_{1}$ for every $t \in \mathbb{R}($ resp. $t \geq 0$ or $t \leq 0)$. A $\sigma$-invariant subset $\Omega_{1} \subset \Omega$ is minimal if it is compact and does not contain properly any other compact $\sigma$-invariant set; or, equivalently, if each one of the two semiorbits of anyone of its elements is dense in it. The continuous flow $(\Omega, \sigma)$ is recurrent or minimal if $\Omega$ itself is minimal.

If the forward semiorbit of a point $\omega_{0} \in \Omega$ is relatively compact, its omega limit set, $\mathcal{O}\left(\omega_{0}\right)$, is given by those points $\omega \in \Omega$ such that $\omega=\lim _{m \rightarrow \infty} \sigma\left(t_{m}, \omega_{0}\right)$ for some sequence $\left(t_{m}\right) \uparrow \infty$. This set is nonempty, compact, connected and forward $\sigma$ invariant. The definition and properties of an alpha limit set $\mathcal{A}\left(\omega_{0}\right)$ are analogous, working now with sequences $\left(t_{m}\right) \downarrow-\infty$.

The summary of the most basic notions required in the present paper is completed with the definitions of invariant and ergodic measures. Let $m$ be a normalized Borel measure on $\Omega$; i.e. a finite regular measure defined on the Borel subsets of $\Omega$ and with $m(\Omega)=1$. The measure $m$ is $\sigma$-invariant if $m\left(\sigma_{t}\left(\Omega_{1}\right)\right)=m\left(\Omega_{1}\right)$ for every Borel subset $\Omega_{1} \subset \Omega$ and every $t \in \mathbb{R}$. If, in addition, $m\left(\Omega_{1}\right)=0$ or $m\left(\Omega_{1}\right)=1$ for every $\sigma$-invariant subset $\Omega_{1} \subset \Omega$, then the measure $m$ is $\sigma$-ergodic. The measure $m$ is concentrated on $\Omega_{1} \subset \Omega$ if $m\left(\Omega_{1}\right)=1$. And the topological support of $m$, Supp $m$, is the complement of the largest open set $O \subset \Omega$ for which $m(O)=0$.

From now on $\Omega$ will indicate a compact metric space and $\sigma: \mathbb{R} \times \Omega \rightarrow \Omega$ a continuous flow, and $\omega \cdot t$ will represent $\sigma(t, \omega)$. Consider the family of linear Hamiltonian systems

$$
\mathbf{z}^{\prime}=\left[\begin{array}{cc}
H_{1}(\omega \cdot t) & H_{3}(\omega \cdot t) \\
H_{2}(\omega \cdot t) & -H_{1}^{T}(\omega \cdot t)
\end{array}\right] \mathbf{z}=H(\omega \cdot t) \mathbf{z}
$$

for $\omega \in \Omega$, where $H$ is a continuous real $2 n \times 2 n$ matrix-valued function on $\Omega$ and $H_{2}$ and $H_{3}$ are $n \times n$ symmetric matrices. Let $U(t, \omega)=\left[\begin{array}{cc}U_{1}(t, \omega) & U_{3}(t, \omega) \\ U_{2}(t, \omega) & U_{4}(t, \omega)\end{array}\right]$ represent the fundamental matrix solution of the system (2.1) for $\omega \in \Omega$ with $U(0, \omega)=I_{2 n}$, which is globally defined. Then the map

$$
\tau_{\mathbb{R}}: \mathbb{R} \times \Omega \times \mathbb{R}^{2 n} \rightarrow \Omega \times \mathbb{R}^{2 n},(t, \omega, \mathbf{z}) \mapsto(\omega \cdot t, U(t, \omega) \mathbf{z})
$$

defines a global continuous flow on $\Omega \times \mathbb{R}^{2 n}$, of linear skew-product type: it preserves the flow on $\Omega$, which can be considered as the base of the bundle $\Omega \times \mathbb{R}^{2 n}$; and it is linear on the fiber component.

Frequently, a family of this type comes from a single nonautonomous Hamiltonian system $\mathbf{z}^{\prime}=H_{0}(t) \mathbf{z}$ by means of the well known Bebutov construction: if $H_{0}$ is 
bounded and uniformly continuous on $\mathbb{R}$, then its hull $\Omega$, defined as $\Omega=\operatorname{cls}\left\{H_{t}, t \in\right.$ $\mathbb{R}\}$ (where $H_{t}(s)=H_{0}(t+s)$ and the closure is taken in the compact-open topology), is a compact metric space and the time-translation defines a continuous flow $\sigma$ on it. The base space $\Omega$ can hence be understood as the space in which the nonautonomous law varies with respect to time. Under additional recurrence properties on $H_{0}$, the base flow is minimal. This is the case if $H_{0}$ is almost periodic or almost automorphic. Weaker conditions on $H_{0}$ may provide a non minimal hull, which can contain different minimal subsets. In some of these cases the solutions of the different linear Hamiltonian systems of the family may show a significatively different qualitative behavior.

2.1. The Lagrangian flow. Recall that two vectors $\mathbf{z}$ and $\mathbf{w}$ in $\mathbb{R}^{2 n}$ are are isotropic if $\mathbf{z}^{T} J \mathbf{w}=0$, where $J=\left[\begin{array}{cc}0_{n} & -I_{n} \\ I_{n} & 0_{n}\end{array}\right]$. Any linear subspace $l \subset \mathbb{R}^{2 n}$ whose vectors are pairwise isotropic satisfies $\operatorname{dim} l \leq n$, since $l$ is contained in the Euclidean subspace orthogonal to $J \cdot l=\{J \mathbf{z} \mid \mathbf{z} \in l\}$. An $n$-dimensional linear subspace $l \subset \mathbb{R}^{2 n}$ is a (real) Lagrange plane if $\mathbf{z}^{T} J \mathbf{w}=0$ for all $\mathbf{z}$ and $\mathbf{w}$ in $l$. The space $\mathcal{L}_{\mathbb{R}}$ of all real Lagrange planes of $\mathbb{R}^{2 n}$ is a compact orientable manifold of dimension $n(n+1) / 2$ : see Matsushima [21] and Mishchenko et al. [22]. An element $l$ of $\mathcal{L}_{\mathbb{R}}$ can be represented by a $2 n \times n$ real matrix $\left[\begin{array}{l}L_{1} \\ L_{2}\end{array}\right]$ of range $n$ with $L_{1}^{T} L_{2}=L_{2}^{T} L_{1}$. We will write $l \equiv\left[\begin{array}{l}L_{1} \\ L_{2}\end{array}\right]$ in what follows. The representation means that the column vectors form the basis of the Lagrange subspace: $\left[\begin{array}{l}L_{1} \\ L_{2}\end{array}\right]$ and $\left[\begin{array}{l}G_{1} \\ G_{2}\end{array}\right]$ represent the same Lagrange plane if and only if there is a nonsingular $n \times n$ real matrix $P$ such that $L_{1}=G_{1} P$ and $L_{2}=G_{2} P$.

The matrix-valued function $H$ belongs to the symplectic Lie algebra $\mathfrak{s p}(n, \mathbb{R})=$ $\left\{G \in \mathbb{M}_{2 n \times 2 n}(\mathbb{R}) \mid G^{T} J+J G=0_{2 n}\right\}$, which implies that, for each $t \in \mathbb{R}$ and $\omega \in \Omega$, $U(t, \omega)$ lies in the symplectic group $S p(n, \mathbb{R})=\left\{V \in \mathbb{M}_{2 n \times 2 n}(\mathbb{R}) \mid V^{T} J V=J\right\}$ : the derivative of $U^{T}(t, \omega) J U(t, \omega)$ is the $2 n \times 2 n$ zero matrix $0_{2 n}$ for all $\omega \in \Omega$ and $t \in \mathbb{R}$. As a consequence of this fact, if $U(t, \omega) \cdot l$ represents the linear space $\{U(t, \omega) \mathbf{z} \mid \mathbf{z} \in l\}$, then $U(t, \omega) \cdot l$ is a new Lagrange plane for all $t \in \Omega$ and $\omega \in \Omega$ : it has dimension $n$ since $U(t, \omega)$ defines an isomorphism on $\mathbb{R}^{2 n}$; and, if $\mathbf{z}$ and $\mathbf{w}$ belong to $l$, then $\mathbf{z}^{T} U^{T}(t, \omega) J U(t, \omega) \mathbf{w}=\mathbf{z}^{T} J \mathbf{w}=0$. This property implies that the map

$$
\tau: \mathbb{R} \times \Omega \times \mathcal{L}_{\mathbb{R}} \rightarrow \Omega \times \mathcal{L}_{\mathbb{R}},(t, \omega, l) \mapsto(\omega \cdot t, U(t, \omega) \cdot l)
$$

defines a global continuous skew-product flow on $\mathcal{K}_{\mathbb{R}}=\Omega \times \mathcal{L}_{\mathbb{R}}$. In addition, if $l \equiv\left[\begin{array}{l}L_{1} \\ L_{2}\end{array}\right]$, then $U(t, \omega) \cdot l \equiv U(t, \omega)\left[\begin{array}{l}L_{1} \\ L_{2}\end{array}\right]=\left[\begin{array}{l}U_{1}(t, \omega) L_{1}+U_{3}(t, \omega) L_{2} \\ U_{2}(t, \omega) L_{1}+U_{4}(t, \omega) L_{2}\end{array}\right]$.

2.2. The Grassmannian flows. Let $W$ be a $d$-dimensional linear subspace of $\mathbb{R}^{2 n}$. Given $k \in\{0,1, \ldots, d\}$, let $\mathcal{G}_{k}(W)$ represent the set of the $k$-dimensional subspaces of $W$. The set $\mathcal{G}_{k}(W)$ can be identified with the homogeneous space of left cosets $G L(d, \mathbb{R}) / \widetilde{\mathcal{H}}$, where $\widetilde{\mathcal{H}}$ is the closed Lie subgroup of $G L(d, \mathbb{R})$ given by the matrices of the form $\left[\begin{array}{ll}A & * \\ 0 & B\end{array}\right]$ for $A \in G L(k, \mathbb{R})$ and $B \in G L(d-k, \mathbb{R})$. Here * represents any $k \times(d-k)$ matrix and 0 represents the zero $(d-k) \times k$ matrix. With this identification, which provides $\mathcal{G}_{k}(W)$ with a differentiable structure, $\mathcal{G}_{k}(W)$ is the Grassmannian manifold of the $k$-dimensional linear subspaces of $W$. This manifold can be also identified with $S O(d, \mathbb{R}) / \mathcal{H}$, where $\mathcal{H}$ is the closed subgroup of $S O(d, \mathbb{R})$ given by the matrices of the form $\left[\begin{array}{ll}A & 0 \\ 0 & B\end{array}\right]$ for $A \in O(k, \mathbb{R}), B \in O(d-k, \mathbb{R})$ and $\operatorname{det} A \cdot \operatorname{det} B=1$. Hence $\mathcal{G}_{k}(W)$ is a compact and connected manifold, which 
agrees with the real projective line if $k=1$. The reader is referred to [21] for the proofs of these properties.

It is clear that the family (2.1) defines a real continuous flow $\tau_{k}$ on $\Omega \times \mathcal{G}_{k}\left(\mathbb{R}^{2 n}\right)$, sending $(\omega, w)$ in time $t$ to $(\omega \cdot t, U(t, \omega) \cdot w)$, where $U(t, \omega) \cdot w=\{U(t, \omega) \mathbf{z} \mid \mathbf{z} \in w\}$ : $\operatorname{dim}(U(t, \omega) \cdot w)=\operatorname{dim} w$, since $U(t, \omega)$ defines an isomorphism of $\mathbb{R}^{2 n}$ for any $t \in \mathbb{R}$ and $\omega \in \Omega$. Note that $\mathcal{K}_{\mathbb{R}}=\Omega \times \mathcal{L}_{\mathbb{R}}$ can be understood as a closed $\tau_{n}$-invariant subset of $\Omega \times \mathcal{G}_{n}\left(\mathbb{R}^{2 n}\right)$, and that $\left.\tau_{n}\right|_{\mathcal{K}_{\mathbb{R}}}$ agrees with the flow $\tau$ defined by $(2.3)$.

2.3. The Riccati flow. Consider the open and dense subset $\mathcal{D}$ of $\mathcal{L}_{\mathbb{R}}$ defined by

$$
\mathcal{D}=\left\{l \in \mathcal{L}_{\mathbb{R}} \mid l \equiv\left[\begin{array}{c}
I_{n} \\
M
\end{array}\right]\right\} .
$$

Obviously, each $l \in \mathcal{D}$ admits a unique representation of the form $\left[\begin{array}{l}I_{n} \\ M\end{array}\right]$, and the $n \times n$ matrix $M$ has to be symmetric: $M \in \mathbb{S}_{n}(\mathbb{R})$. In addition, $l \equiv\left[\begin{array}{l}L_{1} \\ L_{2}\end{array}\right]$ belongs to $\mathcal{D}$ if and only if $\operatorname{det} L_{1} \neq 0$, in which case $M=L_{2} L_{1}^{-1}$. This means that $\mathcal{D}$ is the complement in $\mathcal{L}_{\mathbb{R}}$ of the vertical Maslov cycle $\mathcal{C}$ defined as

$$
\mathcal{C}=\left\{l \in \mathcal{L}_{\mathbb{R}} \mid \operatorname{dim}\left(l \cap l_{0}\right) \geq 1\right\},
$$

where $l_{0}$ is the Lagrange plane generated by the $n$ last coordinate vectors, that is, $l_{0} \equiv\left[\begin{array}{l}0_{n} \\ I_{n}\end{array}\right]$. By continuous dependence with respect to initial data, if $\omega \in \Omega$ and $l \in \mathcal{D}$, then $U(t, \omega) \cdot l \in \mathcal{D}$ for $t$ close enough to zero: if $l \equiv\left[\begin{array}{c}I_{n} \\ M_{0}\end{array}\right]$, then $U(t, \omega) \cdot l$ can be represented by $\left[\begin{array}{c}I_{n} \\ M\left(t, \omega, M_{0}\right)\end{array}\right]$ as long as $\operatorname{det}\left(U_{1}(t, \omega)+U_{3}(t, \omega) M_{0}\right) \neq 0$. Since $M\left(t, \omega, M_{0}\right)=L_{2}(t, \omega) L_{1}(t, \omega)^{-1}$, where $\left[\begin{array}{l}L_{1}(t, \omega) \\ L_{2}(t, \omega)\end{array}\right]$ is the $2 n \times n$ matrix-valued solution of (2.1) with initial data $\left[\begin{array}{c}I_{n} \\ M_{0}\end{array}\right]$, it follows that $M\left(t, \omega, M_{0}\right)$ is the solution of the Riccati equation

$$
M^{\prime}=-M H_{3}(\omega \cdot t) M-M H_{1}(\omega \cdot t)-H_{1}^{T}(\omega \cdot t) M+H_{2}(\omega \cdot t)
$$

with $M\left(0, \omega, M_{0}\right)=M_{0}$. These facts imply that the family of equations (2.5) defines a local continuous skew-product flow

$$
\tau_{s}: \mathbb{R} \times \Omega \times \mathbb{S}_{n}(\mathbb{R}) \rightarrow \Omega \times \mathbb{S}_{n}(\mathbb{R}),\left(t, \omega, M_{0}\right) \mapsto\left(\omega \cdot t, M\left(t, \omega, M_{0}\right)\right),
$$

where, again, $M\left(t, \omega, M_{0}\right)$ solves $(2.5)$ and satisfies $M\left(0, \omega, M_{0}\right)=M_{0}$.

By identifying $\mathcal{D}$ with the linear space $\mathbb{S}_{n}(\mathbb{R}), \tau_{s}$ can be also considered as a local skew-product flow on $\Omega \times \mathcal{D}$. Note that this flow is closely related to the restriction of the flow $\tau$ given by $(2.3)$ to $\Omega \times \mathcal{D} \subset \mathcal{K}_{\mathbb{R}}$ : if $l \equiv\left[\begin{array}{l}I_{n} \\ M_{0}\end{array}\right]$, then $U(t, \omega) \cdot l \equiv$ $\left[\begin{array}{c}I_{n} \\ M\left(t, \omega, M_{0}\right)\end{array}\right]$ as long as the solution $M\left(t, \omega, M_{0}\right)$ exists. It is important to emphasize the fact that, if $l \equiv\left[\begin{array}{l}L_{1} \\ L_{2}\end{array}\right]$ belongs to $\mathcal{D}$, then $U(t, \omega) \cdot l$ belongs to $\mathcal{D}$ as long as $\operatorname{det}\left(U_{1}(t, \omega) L_{1}+U_{3}(t, \omega) L_{2}\right) \neq 0$, which is independent of the representation chosen for $l$. This condition determines then the maximal interval of definition of any solution of $(2.5)$.

2.4. Exponential dichotomy. The next step consists in recalling the definition of exponential dichotomy (see also Johnson [10] and Johnson et al. [16] for more details). The Euclidean norm in $\mathbb{R}^{2 n}$ is fixed and represented by $\|\cdot\|$.

Definition 2.1. The family of systems (2.1) has an exponential dichotomy over $\Omega$ if there exist two positive constants $\eta$ and $\beta$ and a splitting $\Omega \times \mathbb{R}^{2 n}=L^{+} \oplus L^{-}$ of the complex bundle into the Whitney sum of two closed subbundles which are invariant under the flow $\tau_{\mathbb{R}}$ given on $\Omega \times \mathbb{R}^{2 n}$ by (2.2), with the following properties: 
(i) $\|U(t, \omega) \mathbf{z}\| \leq \eta e^{-\beta t}\|\mathbf{z}\| \quad$ for every $t \geq 0$ and $(\omega, \mathbf{z}) \in L^{+}$, and

(ii) $\|U(t, \omega) \mathbf{z}\| \leq \eta e^{\beta t}\|\mathbf{z}\| \quad$ for every $t \leq 0$ and $(\omega, \mathbf{z}) \in L^{-}$.

Proposition 2.2. Suppose that the family (2.1) has an exponential dichotomy over $\Omega$ and let $\Omega \times \mathbb{R}^{2 n}=L^{+} \oplus L^{-}$be the corresponding decomposition. Then, this decomposition is unique and, for each $\omega \in \Omega$, the fibers

$$
l^{ \pm}(\omega)=\left\{\mathbf{z} \mid(\omega, \mathbf{z}) \in L^{ \pm}\right\}
$$

are real Lagrange planes which vary continuously with respect to $\omega$. In particular, the subbundles $L^{ \pm}$are globally $n$-dimensional. In addition, $U(t, \omega) \cdot l^{ \pm}(\omega)=l^{ \pm}(\omega \cdot t)$ for all $t \in \mathbb{R}$ and $\omega \in \Omega$. That is, the sets $\mathcal{L}^{ \pm}=\left\{\left(\omega, l^{ \pm}(\omega)\right) \mid \omega \in \Omega\right\} \subset \mathcal{K}_{\mathbb{R}}$ are $\tau$-invariant, with $\tau$ given by $(2.3): \tau\left(t, \omega, l^{ \pm}(\omega)\right)=\left(\omega \cdot t, l^{ \pm}(\omega \cdot t)\right)$.

\section{Abnormal Linear Hamiltonian Systems}

This section is devoted to a study of the order of abnormality of the family of linear Hamiltonian systems (2.1). Recall that the system (2.1) corresponding to $\omega$ is identically normal on $\mathbb{R}$ if, for every nonzero solution $\left[\begin{array}{l}\mathbf{z}_{1}(t, \omega) \\ \mathbf{z}_{2}(t, \omega)\end{array}\right]$, the vector $\mathbf{z}_{1}(t, \omega)$ does not vanish identically on any nondegenerate interval.

Using a standard notation from Reid [25], for a fixed $\omega \in \Omega$ we denote by $\Lambda_{\omega}([a, \infty))$ the subspace of $n$-dimensional vector-valued functions $\mathbf{z}_{2}(t, \omega)$ which satisfy the equations $\mathbf{z}_{2}^{\prime}=-H_{1}^{T}(\omega \cdot t) \mathbf{z}_{2}, H_{3}(\omega \cdot t) \mathbf{z}_{2}=0$ on $[a, \infty)$, that is, $\left[\begin{array}{c}0 \\ \mathbf{z}_{2}(t, \omega)\end{array}\right]$ is a solution of $(2.1)$ on $[a, \infty)$. Let $d_{\omega}([a, \infty))$ be the dimension of this finitedimensional subspace. Obviously $d_{\omega}([a, \infty)) \leq n$ and it is not hard to check (see also [25]) that it is a nondecreasing and piecewise constant function in $a$ with at most $n$ points of discontinuity, at which it is right-continuous. Hence, there is an $\alpha(\omega)$ such that

$$
d_{\omega}([\alpha(\omega), \infty))=d_{\omega}([t, \infty)) \quad \text { for each } t \geq \alpha(\omega) .
$$

We will denote this quantity by $d^{+}(\omega)$, the index of abnormality at $+\infty$. The system (2.1) is said to be abnormal at $+\infty$ if $d^{+}(\omega)>0$, which means that it has at least one nonzero solution of the form $\left[\begin{array}{c}0 \\ \mathbf{z}_{2}(t, \omega)\end{array}\right]$ in a positive half-line. We refer the reader to Reid [26] and Kratz [18] for a study of abnormal systems. Reid [25] and Šepitka-Šimon Hilscher [29, 30] generalize the concept and theory of principal solutions to this context.

In a analogous way we define $\Lambda_{\omega}((-\infty, b])$ and $d_{\omega}((-\infty, b])=\operatorname{dim} \Lambda_{\omega}((-\infty, b])$. Therefore, there exists $\beta(\omega)$ such that

$$
d_{\omega}((-\infty, \beta(\omega)])=d_{\omega}((-\infty, t])=d^{-}(\omega) \quad \text { for each } t \leq \beta(\omega),
$$

and the system (2.1) is abnormal at $-\infty$ if, $d^{-}(\omega)$, the index of abnormality at $-\infty$, satisfies $d^{-}(\omega)>0$, which means that it has at least one nonzero solution of the form $\left[\begin{array}{c}0 \\ \mathbf{z}_{2}(t, \omega)\end{array}\right]$ in a negative half-line.

Finally, we will denote by $\Lambda_{\omega}(\mathbb{R})$ the subspace of solutions of the system $(2.1)$ of the form $\left[\begin{array}{c}0 \\ \mathbf{z}_{2}(t, \omega)\end{array}\right]$ in $\mathbb{R}$ and by $d(\omega)=\operatorname{dim} \Lambda_{\omega}(\mathbb{R})$ the dimension of this finitedimensional subspace, called the index or order of abnormality. The system (2.1) is abnormal if $d(\omega)>0$. Notice that $d(\omega) \leq \min \left(d^{+}(\omega), d^{-}(\omega)\right)$.

When we consider all the points $\omega \in \Omega$, i.e., all the systems in the family (2.1), the maximum and the minimum number of linearly independent solutions of the 
form $\left[\begin{array}{c}0 \\ \mathbf{z}_{2}(t)\end{array}\right]$ in a positive half-line, a negative half-line or in $\mathbb{R}$ will be denoted by $d_{M}^{+}, d_{m}^{+}, d_{M}^{-}, d_{m}^{-}$and $d_{M}, d_{m}$ respectively, that is,

$$
d_{M}^{ \pm}=\max _{\omega \in \Omega} d^{ \pm}(\omega), d_{M}=\max _{\omega \in \Omega} d(\omega), d_{m}^{ \pm}=\min _{\omega \in \Omega} d^{ \pm}(\omega) \text { and } d_{m}=\min _{\omega \in \Omega} d(\omega) .
$$

The next result collects some important properties of these functions and quantities.

Theorem 3.1. $\quad$ (i) The functions $d^{+}, d^{-}$and $d$ are invariant on $\Omega$, that is, $d^{+}(\omega \cdot s)=d^{+}(\omega), d^{-}(\omega \cdot s)=d^{-}(\omega)$ and $d(\omega \cdot s)=d(\omega)$ for all $\omega \in \Omega$ and $s \in \mathbb{R}$.

(ii) If $\omega_{1} \in \mathcal{O}(\omega)$, then $d^{+}(\omega) \leq d\left(\omega_{1}\right)$, and if $\omega_{1} \in \mathcal{A}(\omega)$, then $d^{-}(\omega) \leq d\left(\omega_{1}\right)$.

(iii) If $m_{0}$ is a $\sigma$-ergodic measure on $\Omega$, then $d^{+}, d^{-}$and $d$ are constant and coincide for $m_{0}$-a.e. $\omega \in \Omega$.

(iv) If $\Omega$ is minimal, then $d^{+}(\omega)=d^{-}(\omega)=d(\omega)$ for all $\omega \in \Omega$, i.e., they are constant functions on $\Omega$ and $d_{M}^{ \pm}=d_{m}^{ \pm}=d_{M}=d_{m}$.

(v) $d_{M}=d_{M}^{ \pm}$and there is a minimal subset $\Omega^{*} \subset \Omega$ such that $d(\omega)=d^{ \pm}(\omega)=$ $d_{M}$ for each $\omega \in \Omega^{*}$.

(vi) $d$ is an upper semicontinuous function, the set of its continuity points is an open residual invariant subset $\Omega_{c} \subset \Omega$ in which $d$ is locally constant, and

$$
\left\{\omega \in \Omega \mid d(\omega)=d_{m}\right\} \subset \Omega_{c} .
$$

(vii) If there exists a point $\omega_{0} \in \Omega$ with dense positive and negative semiorbit, then $d_{m}=d_{m}^{ \pm}$and $d\left(\omega_{0}\right)=d^{ \pm}\left(\omega_{0}\right)=d_{m}$. In particular, $d$ is continuous at $\omega_{0}$.

(viii) If $m_{0}$ is a $\sigma$-ergodic measure on $\Omega$ with $\operatorname{Supp} m_{0}=\Omega$, then $d_{m}=d_{m}^{ \pm}$ and there is a subset $\Omega_{1} \subset \Omega$ with full measure $m_{0}\left(\Omega_{1}\right)=1$ such that $d(\omega)=d^{ \pm}(\omega)=d_{m}$ for each $\omega \in \Omega_{1}$. In particular, $m_{0}\left(\Omega_{c}\right)=1$. In addition, in this case $\Omega_{c}=\left\{\omega \in \Omega \mid d(\omega)=d_{m}\right\}$.

Proof. (i) is an easy consequence of the fact that if $\left[\begin{array}{c}0 \\ \mathbf{u}(t)\end{array}\right]$ is a solution of the system $(2.1)$, then $\left[\begin{array}{c}0 \\ \mathbf{u}(t)\end{array}\right]=\left[\begin{array}{c}0 \\ \mathbf{u}(t+s)\end{array}\right]$ is a solution of $\mathbf{z}^{\prime}=H((\omega \cdot s) \cdot t) \mathbf{z}$.

(ii) We will only prove that $d^{+}(\omega) \leq d\left(\omega_{1}\right)$ if $\omega_{1} \in \mathcal{O}(\omega)$ because the other inequality is proved in an analogous way. If $d^{+}(\omega)=0$ the inequality is obvious.

Let $d^{+}(\omega)=k>0$ and consider a $2 n \times k$ matrix solution of $(2.1)\left[\begin{array}{l}G_{1}(t, \omega) \\ G_{2}(t, \omega)\end{array}\right]$ belonging to $\mathcal{G}_{k}\left(\mathbb{R}^{2 n}\right)$ and of the form $\left[\begin{array}{c}0 \\ G_{2}(t, \omega)\end{array}\right]$ for $t \geq \alpha(\omega)$. Since $\omega_{1} \in \mathcal{O}(\omega)$, we take a sequence $\left(t_{n}\right) \uparrow \infty$ with $\omega_{1}=\lim _{n \rightarrow \infty} \omega \cdot t_{n}$. The sequence $\left[\begin{array}{c}0 \\ G_{2}\left(t_{n}, \omega\right)\end{array}\right]$ of $\mathcal{G}_{k}\left(\mathbb{R}^{2 n}\right)$ admits a convergent subsequence $\left[\begin{array}{c}0 \\ G_{2}\left(t_{n_{j}}, \omega\right)\end{array}\right] \rightarrow\left[\begin{array}{c}0 \\ \widetilde{G}_{2}\end{array}\right]$. The continuity of the flow $\tau_{k}$ on $\Omega \times \mathcal{G}_{k}\left(\mathbb{R}^{2 n}\right)$ implies that

$$
\begin{aligned}
U\left(t, \omega_{1}\right)\left[\begin{array}{c}
0 \\
\widetilde{G}_{2}
\end{array}\right] & =\lim _{j \rightarrow \infty} U\left(t, \omega \cdot t_{n_{j}}\right)\left[\begin{array}{c}
0 \\
G_{2}\left(t_{n_{j}}, \omega\right)
\end{array}\right]=\lim _{j \rightarrow \infty} U\left(t, \omega \cdot t_{n_{j}}\right) U\left(t_{n_{j}}, \omega\right)\left[\begin{array}{l}
G_{1}(0, \omega) \\
G_{2}(0, \omega)
\end{array}\right] \\
& =\lim _{j \rightarrow \infty} U\left(t+t_{n_{j}}, \omega\right)\left[\begin{array}{l}
G_{1}(0, \omega) \\
G_{2}(0, \omega)
\end{array}\right]=\lim _{j \rightarrow \infty}\left[\begin{array}{l}
G_{1}\left(t+t_{n_{j}}, \omega\right) \\
G_{2}\left(t+t_{n_{j}}, \omega\right)
\end{array}\right],
\end{aligned}
$$

and hence $U\left(t, \omega_{1}\right)\left[\begin{array}{c}0 \\ \widetilde{G}_{2}\end{array}\right]$ is of the form $\left[\begin{array}{c}0 \\ \left.\widetilde{G}_{2}(t)\right)\end{array}\right]$ for each $t \in \mathbb{R}$ because $t+t_{n_{j}} \geq \alpha(\omega)$ for $n_{j}$ large enough. This shows that $d^{+}(\omega) \leq d\left(\omega_{1}\right)$, as stated.

(iii) From (i) $d^{+}, d^{-}$and $d$ are constant for $m_{0^{-}}$-a.e. $\omega \in \Omega$. In addition, by the Poincaré Recurrence Theorem (see [2]) there is a subset $\Omega_{0}$ of full measure such that $\omega \in \mathcal{O}(\omega) \cap \mathcal{A}(\omega)$ for $\omega \in \Omega_{0}$, and the coincidence of $d, d^{+}$and $d^{-}$in a set of full measure follows from (ii). 
(iv) follows immediately from (ii).

(v) Take $\omega_{0} \in \Omega$ such that $d^{+}\left(\omega_{0}\right)=d_{M}^{+}$. From (ii), we deduce that $d_{M} \leq d_{M}^{+}=$ $d^{+}\left(\omega_{0}\right) \leq d(\omega) \leq d_{M}$ for each $\omega \in \mathcal{O}\left(\omega_{0}\right)$, and hence, $d_{M}=d_{M}^{+}$. Analogously, if we take $\omega_{1} \in \Omega$ with $d^{-}\left(\omega_{1}\right)=d_{M}^{-}$, for each $\omega \in \mathcal{A}\left(\omega_{1}\right)$ we have $d_{M} \leq d_{M}^{-}=d^{-}\left(\omega_{1}\right) \leq$ $d(\omega) \leq d_{M}$ and consequently $d_{M}^{-}=d_{M}$, that is, $d_{M}=d_{M}^{ \pm}$, as asserted. Finally, taking a minimal subset $\Omega^{*} \subset \mathcal{O}\left(\omega_{0}\right)$, from (iv) we conclude that $d(\omega)=d^{ \pm}(\omega)=$ $d_{m}$ for each $\omega \in \Omega^{*}$.

(vi) We have to check that $\lim \sup _{\omega \rightarrow \omega_{0}} d(\omega) \leq d\left(\omega_{0}\right)$ for each $\omega_{0} \in \Omega$. Let $\left(\omega_{k}\right)$ be a sequence with $\lim _{k \rightarrow \infty} \omega_{k}=\omega_{0}$. Since $d\left(\omega_{k}\right) \leq n$ and $d$ takes only integer values, it has a constant subsequence, i.e., $d\left(\omega_{k_{j}}\right)=k_{0}$ for each $j \in \mathbb{N}$.

Therefore, we can take $\left[\begin{array}{l}G_{1}\left(t, \omega_{k_{j}}\right) \\ G_{2}\left(t, \omega_{k_{j}}\right)\end{array}\right]$ a $2 n \times k_{0}$ matrix solution of (2.1) belonging to $\mathcal{G}_{k}\left(\mathbb{R}^{2 n}\right)$ of the form $\left[\begin{array}{c}0 \\ G_{2}\left(t, \omega_{k_{j}}\right)\end{array}\right]=U\left(t, \omega_{k_{j}}\right)\left[\begin{array}{c}0 \\ G_{2}\left(0, \omega_{k_{j}}\right)\end{array}\right]$ for each $t \in \mathbb{R}$, and assume that $\left[\begin{array}{c}0 \\ G_{2}\left(0, \omega_{k_{j}}\right)\end{array}\right] \rightarrow\left[\begin{array}{c}0 \\ G_{2}\end{array}\right]$ as $j \rightarrow \infty$ in $\mathcal{G}_{k_{0}}\left(\mathbb{R}^{2 n}\right)$ (the result is true for an appropriate subsequence, as above). Again, the continuity of the flow $\tau_{k_{0}}$ on $\Omega \times \mathcal{G}_{k_{0}}\left(\mathbb{R}^{2 n}\right)$ implies that

$$
U\left(t, \omega_{0}\right)\left[\begin{array}{c}
0 \\
G_{2}
\end{array}\right]=\lim _{j \rightarrow \infty} U\left(t, \omega_{k_{j}}\right)\left[\begin{array}{c}
0 \\
G_{2}\left(0, \omega_{k_{j}}\right)
\end{array}\right]=\lim _{j \rightarrow \infty}\left[\begin{array}{c}
0 \\
G_{2}\left(t, \omega_{k_{j}}\right)
\end{array}\right],
$$

is hence of the form $\left[\begin{array}{c}0 \\ \left.G_{2}(t)\right)\end{array}\right]$, and therefore $k_{0} \leq d\left(\omega_{0}\right)$, which implies that $d$ is upper semicontinuous.

As a consequence, the set $\Omega_{c} \subset \Omega$ of continuity points is an invariant and residual set which is necessarily open because $d$ only takes integer values, and hence for each $\omega \in \Omega_{c}$ there is an open ball $B\left(\omega, \delta_{\omega}\right) \subset \Omega_{c}$ in which $d$ is constant, that is, $d$ is locally constant in $\Omega_{c}$. Finally, we check that each $\omega \in \Omega$ with $d(\omega)=d_{m}$ is a continuity point. Let $\left(\omega_{k}\right)$ be a sequence such that $\lim _{k \rightarrow \infty} \omega_{k}=\omega$. From the definition of $d_{m}$ and the upper semicontinuity of $d$ we deduce that

$$
d_{m} \leq \liminf _{k \rightarrow \infty} d\left(\omega_{k}\right) \leq \limsup _{k \rightarrow \infty} d\left(\omega_{k}\right) \leq d(\omega)=d_{m},
$$

and hence $\lim _{k \rightarrow \infty} d\left(\omega_{k}\right)=d(\omega)$, as stated.

(vii) Since $\mathcal{O}\left(\omega_{0}\right)=\Omega, \mathcal{A}\left(\omega_{0}\right)=\Omega$ and $d(\omega) \leq d^{ \pm}(\omega)$, we deduce from (ii) that $d_{m} \leq d_{m}^{ \pm} \leq d^{ \pm}\left(\omega_{0}\right) \leq d(\omega) \leq d^{ \pm}(\omega)$ for each $\omega \in \Omega$, which in particular implies that $d\left(\omega_{0}\right)=d^{ \pm}\left(\omega_{0}\right)$ and taking the infimum in $\omega \in \Omega$ that $d_{m}=d_{m}^{ \pm}$, as stated.

(viii) From Supp $m_{0}=\Omega$, we deduce the existence of a subset $\Omega_{1} \subset \Omega$ with full measure such that the positive and negative semiorbit of each $\omega \in \Omega_{1}$ is dense in $\Omega$, and the first part of the statement follows from (vii). The invariance of $d$ and the density of one of these trajectories show that, in this case, the set of continuity points coincides with $\left\{\omega \in \Omega \mid d(\omega)=d_{m}\right\}$.

Next, for a fixed $(\omega, l) \in \Omega \times \mathcal{L}_{\mathbb{R}}$, we want to study the number of independent solutions of $(2.1)$ of the form $\left[\begin{array}{c}0 \\ \mathbf{z}_{2}(t, \omega)\end{array}\right]$ in a positive, negative half-line or in the full line, with initial data in the subspace $l$.

First of all notice that if we denote by

$$
\begin{aligned}
\Lambda^{+}(\omega) & =\left\{\mathbf{z}_{0} \in \mathbb{R}^{2 n} \mid U(t, \omega) \mathbf{z}_{0}=\left[\begin{array}{c}
0 \\
\mathbf{z}_{2}(t, \omega)
\end{array}\right] \text { for } t \text { in a positive half-line }\right\} \\
\Lambda^{-}(\omega) & =\left\{\mathbf{z}_{0} \in \mathbb{R}^{2 n} \mid U(t, \omega) \mathbf{z}_{0}=\left[\begin{array}{c}
0 \\
\mathbf{z}_{2}(t, \omega)
\end{array}\right] \text { for } t \text { in a negative half-line }\right\}, \\
\Lambda(\omega) & =\left\{\mathbf{z}_{0} \in \mathbb{R}^{2 n} \mid U(t, \omega) \mathbf{z}_{0}=\left[\begin{array}{c}
0 \\
\mathbf{z}_{2}(t, \omega)
\end{array}\right] \text { for } t \in \mathbb{R}\right\},
\end{aligned}
$$


we have $d^{ \pm}(\omega)=\operatorname{dim}\left(\Lambda^{ \pm}(\omega)\right)$ and $d(\omega)=\operatorname{dim}(\Lambda(\omega))$.

Now we define

$$
\begin{aligned}
& d^{ \pm}: \Omega \times \mathcal{L}_{\mathbb{R}} \rightarrow \quad\{0, \ldots, n\} \quad d: \Omega \times \mathcal{L}_{\mathbb{R}} \rightarrow \quad\{0, \ldots, n\} \\
& (\omega, l) \mapsto \operatorname{dim}\left(\Lambda^{ \pm}(\omega) \cap l\right), \quad(\omega, l) \quad \mapsto \operatorname{dim}(\Lambda(\omega) \cap l) .
\end{aligned}
$$

The next lemma provides a relation between $d(\omega)$ and $d(\omega, l)$ as well as between $d^{ \pm}(\omega)$ and $d^{ \pm}(\omega, l)$.

Lemma 3.2. (i) Let $\omega \in \Omega$ and $k \in\{0, \ldots, d(\omega)\}$. Then there is a Lagrange plane $l \in \mathcal{L}_{\mathbb{R}}$ such that $d(\omega, l)=k$.

(ii) Let $\omega \in \Omega$ and $k \in\left\{0, \ldots, d^{+}(\omega)\right\}$. Then there is a Lagrange plane $l \in \mathcal{L}_{\mathbb{R}}$ such that $d^{+}(\omega, l)=k$.

(iii) Let $\omega \in \Omega$ and $k \in\left\{0, \ldots, d^{-}(\omega)\right\}$. Then there is a Lagrange plane $l \in \mathcal{L}_{\mathbb{R}}$ such that $d^{-}(\omega, l)=k$.

Proof. (i) For $k=0$ it is immediate to check that $d(\omega, l)=0$ for $l \equiv\left[\begin{array}{c}I_{n} \\ 0\end{array}\right]$; and for $k=n$ it is also clear that $d(\omega, l)=n$ for $l \equiv\left[\begin{array}{c}0 \\ I_{n}\end{array}\right]$, so take $1 \leq k<n$. Since there are $d(\omega)$ linearly independent solutions of the form $\left[\begin{array}{c}0 \\ \mathbf{z}_{2}(t, \omega)\end{array}\right]$, we can take $k$ vectors $\mathbf{z}_{0}^{j}=\left[\begin{array}{c}0 \\ \mathbf{z}_{0,2}^{j}\end{array}\right], j=1, \ldots, k$, such that $U(t, \omega) \mathbf{z}_{0}^{j}=\left[\begin{array}{c}0 \\ \mathbf{z}_{2}^{j}(t, \omega)\end{array}\right]$. Then we consider the $k$ dimensional subspace generated by $\mathbf{z}_{0,2}^{1}, \ldots, \mathbf{z}_{0,2}^{k}$ and its orthogonal subspace which has dimension $n-k$. If we take a basis $\left\{\mathbf{w}_{1}, \ldots, \mathbf{w}_{n-k}\right\}$, it is immediate to check that $l \equiv\left[\begin{array}{cccccc}0 & \cdots & 0 & \mathbf{w}_{1} & \cdots & \mathbf{w}_{n-k} \\ \mathbf{z}_{0,2}^{1} & \cdots & \mathbf{z}_{0,2}^{k} & 0 & \cdots & 0\end{array}\right]$ is a Lagrange plane and $d(\omega, l)=k$.

The proof of (ii) (resp. (iii)) is almost analogous to that of (i). The only difference is that now we can take $k$ vectors of this form at a positive time $t_{0}^{+}$(resp. at a negative time $\left.t_{0}^{-}\right)$to define a Lagrange plane $l^{+}\left(\right.$resp. $\left.l^{-}\right)$as above, and we consider $l=U\left(-t_{0}^{+}, \omega \cdot\left(-t_{0}^{+}\right)\right) \cdot l^{+}\left(\right.$resp. $\left.l=U\left(-t_{0}^{-}, \omega \cdot\left(-t_{0}^{+}\right)\right) \cdot l^{-}\right)$to show that $d^{+}(\omega, l)=k$ (resp. $\left.d^{-}(\omega, l)=k\right)$.

As a consequence, the maxima of $d$ and $d^{ \pm}$on $\Omega \times \mathcal{L}_{\mathbb{R}}$ coincide with the maxima of $d$ and $d^{ \pm}$on $\Omega$, as defined in (3.1), that is,

$$
d_{M}=\max _{(\omega, l) \in \Omega \times \mathcal{L}_{\mathbb{R}}} d(\omega, l), \quad d_{M}^{ \pm}=\max _{(\omega, l) \in \Omega \times \mathcal{L}_{\mathbb{R}}} d^{ \pm}(\omega, l),
$$

and the minimum of $d$ on $\Omega \times \mathcal{L}_{\mathbb{R}}$ is 0 .

Proposition 3.3. $\quad$ (i) The functions $d^{+}, d^{-}$and $d$ are invariant in $\Omega \times \mathcal{L}_{\mathbb{R}}$, that is, $d^{+}\left(\tau_{s}(\omega, l)\right)=d^{+}(\omega, l), d^{-}\left(\tau_{s}(\omega, l)\right)=d^{-}(\omega, l)$ and $d\left(\tau_{s}(\omega, l)\right)=d(\omega, l)$ for each $(\omega, l) \in \Omega \times \mathcal{L}_{\mathbb{R}}$ and $s \in \mathbb{R}$.

(ii) If $\left(\omega_{1}, l_{1}\right) \in \mathcal{O}(\omega, l)$, then $d^{+}(\omega, l) \leq d\left(\omega_{1}, l_{1}\right)$, and if $\left(\omega_{1}, l_{1}\right) \in \mathcal{A}(\omega, l)$, then $d^{-}(\omega, l) \leq d\left(\omega_{1}, l_{1}\right)$.

(iii) If $\mu$ is a $\tau$-ergodic measure on $\Omega \times \mathcal{L}_{\mathbb{R}}$, then $d^{+}, d^{-}$and $d$ are constant and coincide for $\mu$-a.e. $(\omega, l) \in \Omega \times \mathcal{L}_{\mathbb{R}}$.

(iv) If $K$ is a minimal set of $\Omega \times \mathcal{L}_{\mathbb{R}}$, then $d^{+}(\omega, l)=d^{-}(\omega, l)=d(\omega, l)=d$ for each $(\omega, l) \in K$, i.e., they are constant functions on $K$.

(v) $d_{M}=d_{M}^{ \pm}$and there is a minimal subset $K^{*} \subset \Omega \times \mathcal{L}_{\mathbb{R}}$ such that $d(\omega, l)=$ $d^{ \pm}(\omega, l)=d_{M}$ for each $(\omega, l) \in K^{*}$.

(vi) $d$ is an upper semicontinuous function on $\Omega \times \mathcal{L}_{\mathbb{R}}$. For each $k \in\left\{0, \ldots, d_{M}\right\}$, the sets

$$
\mathcal{L}_{k}=\left\{(\omega, l) \in \Omega \times \mathcal{L}_{\mathbb{R}} \mid d(\omega, l) \geq k\right\}
$$


are closed, and $\mathcal{L}_{k}-\mathcal{L}_{k+1}=\left\{(\omega, l) \in \Omega \times \mathcal{L}_{\mathbb{R}} \mid d(\omega, l)=k\right\}$ is an open residual and dense set in $\mathcal{L}_{k}$, which coincides with the set of continuity points of $d$ in $\mathcal{L}_{k}$.

Proof. We omit the proofs of (i)-(v) and of the upper semicontinuity of $d$ on $\Omega \times \mathcal{L}_{\mathbb{R}}$ because they are analogous to the corresponding ones in Theorem 3.1.

(vi) From the upper semicontinuity of $d$ it is easily deduced that $\mathcal{L}_{k}$ is closed, and hence $\mathcal{L}_{k}-\mathcal{L}_{k+1}$ is open in $\mathcal{L}_{k}$. To check the density, assume that $k<n$ (since the case $k=n$ is trivial), and take $(\omega, l) \in \mathcal{L}_{\mathbb{R}}$ with $d(\omega, l)=k+1$. We consider two possible cases depending on the expression of $l$ :

1. $l \equiv\left[\begin{array}{cccccc}0 & \cdots & 0 & \mathbf{z}_{1}^{k+2} & \cdots & \mathbf{z}_{1}^{n} \\ \mathbf{z}_{2}^{1} & \cdots & \mathbf{z}_{2}^{k+1} & 0 & \cdots & 0\end{array}\right]$. Denote by $S=\left\langle\mathbf{z}_{2}^{1}, \ldots, \mathbf{z}_{2}^{k}\right\rangle$ the subspace generated by the vectors $\mathbf{z}_{2}^{1}, \ldots, \mathbf{z}_{2}^{k}$ (the null space if $k=0$ ) and choose a vector $\mathbf{z}_{1}$ orthogonal to $S$ and which is not a linear combination of the vectors $\mathbf{z}_{1}^{k+2}, \ldots, \mathbf{z}_{1}^{n}$. Then the subspace $l_{\varepsilon} \equiv\left[\begin{array}{cccccc}0 & \cdots & \varepsilon \widetilde{\mathbf{z}}_{1} & \mathbf{z}_{1}^{k+2} & \cdots & \mathbf{z}_{1}^{n} \\ \mathbf{z}_{2}^{1} & \cdots & \mathbf{z}_{2}^{k+1} & 0 & \cdots & 0\end{array}\right]$ is a Lagrange plane for each $\varepsilon$, satisfies $d\left(\omega, l_{\varepsilon}\right)=k$, i.e., belongs to $\mathcal{L}_{k}-\mathcal{L}_{k+1}$, and $l_{\varepsilon}$ converges to $l$ as $\varepsilon$ goes to 0 .

2. $l \equiv\left[\begin{array}{ccccccccc}0 & \cdots & 0 & \mathbf{z}_{1}^{k+2} & \cdots & \mathbf{z}_{1}^{d} & \mathbf{z}_{1}^{d+1} & \cdots & \mathbf{z}_{1}^{n} \\ \mathbf{z}_{2}^{1} & \cdots & \mathbf{z}_{2}^{k+1} & \mathbf{z}_{2}^{k+2} & \cdots & \mathbf{z}_{2}^{d} & 0 & \cdots & 0\end{array}\right]$ where $k+2 \leq d \leq n, \mathbf{z}_{1}^{j} \neq 0$ for each $j=k+2, \ldots, n$ and the vectors $\mathbf{z}_{2}^{1}, \ldots, \mathbf{z}_{2}^{d}$ are linearly independent. If $d<n$ we choose a vector $\widetilde{\mathbf{z}}_{1} \neq 0$ orthogonal to the subspace $\left\langle\mathbf{z}_{2}^{1}, \ldots \mathbf{z}_{2}^{k}, \mathbf{z}_{2}^{k+2}, \ldots, \mathbf{z}_{2}^{d}\right\rangle$ and which is not a linear combination of the vectors $\mathbf{z}_{1}^{d+1}, \ldots, \mathbf{z}_{1}^{n}$. If $d=n$ we choose a vector $\widetilde{\mathbf{z}}_{1} \neq 0$ orthogonal to the subspace $\left\langle\mathbf{z}_{2}^{1}, \ldots \mathbf{z}_{2}^{k}, \mathbf{z}_{2}^{k+2} \ldots, \mathbf{z}_{2}^{d}\right\rangle$. We claim that, in both cases, $\widetilde{\mathbf{z}}_{1}$ is not a linear combination of the vectors $\mathbf{z}_{1}^{k+2}, \ldots, \mathbf{z}_{1}^{n}$. Assume on the contrary this possibility, that is, $\widetilde{\mathbf{z}}_{1}=\sum_{j=k+2}^{n} \lambda_{j} \mathbf{z}_{1}^{j}$ with $\lambda_{j} \neq 0$ for at least one $j \in\{k+2, \ldots, d\}$. If $\lambda_{k+2} \neq 0$ (a similar argument would apply for any

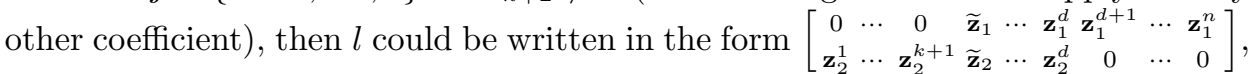
which implies that $\widetilde{\mathbf{z}}_{1}$ is also orthogonal to $\mathbf{z}_{2}^{k+1}$. Hence, $\widetilde{\mathbf{z}}_{1}$ belongs to the subspace orthogonal to $\left\langle\mathbf{z}_{2}^{1}, \ldots, \mathbf{z}_{2}^{d}\right\rangle$, which is the subspace $\left\langle\mathbf{z}_{1}^{d+1}, \ldots, \mathbf{z}_{2}^{n}\right\rangle$ if $d<n$, and the null space if $d=n$, contradicting the choice of $\widetilde{\mathbf{z}}_{1}$ in both cases. Therefore, the

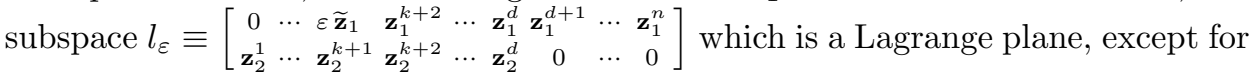
at most a finite number of $\varepsilon$, satisfies $d\left(\omega, l_{\varepsilon}\right)=k$. Hence, we can take a sequence $\left(\varepsilon_{j}\right)$ going to 0 such that $l_{\varepsilon_{j}}$ is a Lagrange plane satisfying $d\left(\omega, l_{\varepsilon_{j}}\right)=k$, i.e., belongs to $\mathcal{L}_{k}-\mathcal{L}_{k+1}$ and $l_{\varepsilon_{j}}$ converges to $l$ as $\varepsilon_{j}$ goes to 0 .

Similar arguments can be extended to the cases $d(\omega, l)>k+1$, which shows the asserted density of the set $\mathcal{L}_{k}-\mathcal{L}_{k+1}$ in $\mathcal{L}_{k}$. As a consequence, the set of continuity points of $d$ in $\mathcal{L}_{k}$, which is a residual and invariant subset, is necessarily contained in $\mathcal{L}_{k}-\mathcal{L}_{k+1}$. Finally, we check that each $(\omega, l) \in \mathcal{L}_{k}$ with $d(\omega, l)=k$ is a continuity point, which finishes the proof. Let $\left(\omega_{j}, l_{j}\right)$ be a sequence of $\mathcal{L}_{k}$ such that $\lim _{j \rightarrow \infty}\left(\omega_{j}, l_{j}\right)=(\omega, l)$. From the definition of $\mathcal{L}_{k}$ and the upper semicontinuity of $d$ we deduce that

$$
k \leq \liminf _{j \rightarrow \infty} d\left(\omega_{j}, l_{j}\right) \leq \limsup _{j \rightarrow \infty} d\left(\omega_{j}, l_{j}\right) \leq d(\omega, l)=k,
$$

and hence $\lim _{j \rightarrow \infty} d\left(\omega, l_{j}\right)=k$ as stated. 
Assume now that the family (2.1) admits an exponential dichotomy over $\Omega$ and let $\Omega \times \mathbb{R}^{2 n}=L^{+} \oplus L^{-}$be the corresponding decomposition with associated Lagrange planes $l^{ \pm}(\omega)=\left\{\mathbf{z} \mid(\omega, \mathbf{z}) \in L^{ \pm}\right\}$. We define the functions

$$
\widetilde{d}^{ \pm}: \Omega \rightarrow\{0, \ldots, n\}, \quad \omega \mapsto \widetilde{d}^{ \pm}(\omega)=d\left(\omega, l^{ \pm}(\omega)\right) .
$$

Is it not hard to check that these functions satisfy analogous properties to the ones stated in Theorem 3.1 for the function $d$ but we omit the statements and corresponding proofs. The next result relates $\widetilde{d}^{ \pm}$with $d$ and in particular proves that if $\omega \in \mathcal{O}(\omega) \cup \mathcal{A}(\omega)$, then the number of linearly independent solutions of (2.1) of the form $\left[\begin{array}{c}0 \\ \mathbf{z}_{2}(t, \omega)\end{array}\right]$ can be calculated in terms of the number of linearly independent solutions of this form which are bounded as $t$ goes to $\pm \infty$, or what is the same thing, have initial data in the subspaces $l^{+}(\omega)$ and $l^{-}(\omega)$.

Theorem 3.4. Assume that the family (2.1) has an exponential dichotomy over $\Omega$. Then

(i) if $\omega \in \mathcal{O}(\omega) \cup \mathcal{A}(\omega)$, then $d(\omega)=\widetilde{d}^{+}(\omega)+\widetilde{d}^{-}(\omega)$;

(ii) if $m_{0}$ is a $\sigma$-ergodic measure, then there are constants $\widetilde{d}^{+}, \widetilde{d}^{-}$, and $d$ such that $d=\widetilde{d}^{+}+\widetilde{d}^{-}$and $\widetilde{d}^{ \pm}(\omega)=\widetilde{d}^{ \pm}, d(\omega)=d$ for $m_{0}$-a.e. $\omega \in \Omega$;

(iii) if Supp $m_{0}=\Omega$, then the equalities of (ii) hold in the open residual invariant set of full measure $\left\{\omega \in \Omega \mid d(\omega)=d_{m}\right\}$;

(iv) if $\Omega$ is minimal, then the equalities of (ii) hold for all $\omega \in \Omega$.

Proof. (i) The result is obviously true if $d(\omega)=0$, since $0 \leq \widetilde{d}^{ \pm}(\omega) \leq d(\omega)$. Assume that $d(\omega)=d>0$, and call $k=\operatorname{dim}\left(\Lambda(\omega) \cap l^{+}(\omega)\right) \geq 0$. Take $d$ linearly independent vectors $\mathbf{z}_{1}=\left[\begin{array}{c}0 \\ \mathbf{z}_{1,2}\end{array}\right], \ldots, \mathbf{z}_{d}=\left[\begin{array}{c}0 \\ \mathbf{z}_{d, 2}\end{array}\right]$ in $\Lambda(\omega)$ such that $\mathbf{z}_{j} \in l^{+}(\omega)$ for $j=1, \ldots, k$; decompose $\mathbf{z}_{j}=\mathbf{z}_{j}^{+}+\mathbf{z}_{j}^{-}$with $\mathbf{z}_{j}^{ \pm} \in l^{ \pm}(\omega)$ for $j=1, \ldots, d$, and note that $\mathbf{z}_{j}^{-}=0$ for $j=1, \ldots, k$. Let $U(t, \omega) \mathbf{z}_{j}=\left[\begin{array}{c}0 \\ \mathbf{z}_{2, j}(t, \omega)\end{array}\right]$ be the corresponding solutions for $j=1, \ldots, k$. Now we assume that $\omega \in \mathcal{O}(\omega)$, take a sequence $\left(t_{m}\right) \uparrow \infty$ with $\lim _{m \rightarrow \infty} \omega \cdot t_{m}=\omega$, and we suppose without restriction that

$$
\begin{aligned}
\left\langle\widetilde{\mathbf{z}}_{1}, \ldots, \widetilde{\mathbf{z}}_{k}\right\rangle & =\lim _{m \rightarrow \infty} U\left(t_{m}, \omega\right) \cdot\left\langle\mathbf{z}_{1}, \ldots, \mathbf{z}_{k}\right\rangle \quad \text { in } \mathcal{G}_{k}\left(\mathbb{R}^{2 n}\right), \\
\left\langle\widetilde{\mathbf{z}}_{k+1}, \ldots, \widetilde{\mathbf{z}}_{d}\right\rangle & =\lim _{m \rightarrow \infty} U\left(t_{m}, \omega\right) \cdot\left\langle\mathbf{z}_{k+1}, \ldots, \mathbf{z}_{n}\right\rangle \text { in } \mathcal{G}_{d-k}\left(\mathbb{R}^{2 n}\right) .
\end{aligned}
$$

The arguments of Theorem 3.1(ii) of [16] allow us to prove that

$$
\left\langle\widetilde{\mathbf{z}}_{1}, \ldots, \widetilde{\mathbf{z}}_{k}\right\rangle \in \mathcal{G}_{k}\left(l^{+}(\omega)\right) \text { and }\left\langle\widetilde{\mathbf{z}}_{k+1}, \ldots, \widetilde{\mathbf{z}}_{d}\right\rangle \in \mathcal{G}_{d-k}\left(l^{-}(\omega)\right) .
$$

Repeating now the argument of Theorem 3.1(ii) shows that $U(t, \omega) \widetilde{\mathbf{z}}_{j}$ is of the form $\left[\begin{array}{c}0 \\ \widetilde{\mathbf{z}}_{2, j}(t, \omega)\end{array}\right]$ for each $j=1, \ldots, d$. Therefore, $\widetilde{d}^{+}(\omega)=d\left(\omega, l^{+}(\omega)\right) \geq k$ and $\widetilde{d}^{-}(\omega)=d\left(\omega, l^{-}(\omega)\right) \geq d-k$. Since

$$
\begin{aligned}
\tilde{d}^{+}(\omega)+\tilde{d}^{-}(\omega) & =\operatorname{dim}\left(\Lambda(\omega) \cap l^{+}(\omega)\right)+\operatorname{dim}\left(\Lambda(\omega) \cap l^{-}(\omega)\right) \\
& \leq \operatorname{dim}\left(\Lambda(\omega) \cap\left(l^{+}(\omega) \oplus l^{-}(\omega)\right)\right)=d(\omega),
\end{aligned}
$$

we conclude that $d(\omega)=\widetilde{d}^{+}(\omega)+\widetilde{d}^{-}(\omega)$, as stated. We omit the proof of the case $\omega \in \mathcal{A}(\omega)$, which is analogous.

(ii) From Theorem 3.1(iii) and the corresponding result for $\widetilde{d}^{ \pm}$, the three functions $d, \widetilde{d}^{+}$and $\widetilde{d}^{-}$are constant for $m_{0}$-a.e. $\omega \in \Omega$; in addition, by the Poincaré Recurrence Theorem there is a subset $\Omega_{0}$ of full measure such that $\omega \in \mathcal{O}(\omega) \cap \mathcal{A}(\omega)$ for all $\omega \in \Omega_{0}$, which together with (i) prove the statement. 
(iii) From Theorem 3.1(vi)\&(viii) and the corresponding properties for $\widetilde{d}^{+}$and $\widetilde{d}^{-}$, the intersection of the sets of continuity points for $d, \widetilde{d}^{+}$and $\widetilde{d}^{-}$provides an open residual invariant set of full measure for which the equalities of (ii) hold. To finish the proof of the statement, notice that if $d(\omega)=d_{m}$, from $\widetilde{d}^{+}(\omega)+\widetilde{d}^{-}(\omega) \leq d(\omega)$ we deduce that $\widetilde{d}^{+}(\omega)$ and $\widetilde{d}^{-}(\omega)$ also attain the minimum at $\omega$ and the previous intersection coincide with $\left\{\omega \in \Omega \mid d(\omega)=d_{m}\right\}$, as stated.

(iv) From Theorem 3.1(iv) and the corresponding result for $\widetilde{d}^{+}$and $\widetilde{d}^{-}$we deduce they are constant functions on $\Omega$. In addition, since $\Omega$ is minimal every trajectory is dense and the result follows from (i).

3.1. Proper focal points and rotation number. As we have explained, the abnormality of the Hamiltonian system (2.1) is measured by the existence of nonzero solutions of the form $\left[\begin{array}{c}0 \\ \mathbf{z}_{2}(t, \omega)\end{array}\right]$ in a half-line. Given a conjoined basis, i.e., a $2 n \times n$ matrix solution $U(t, \omega) \cdot l \equiv U(t, \omega)\left[\begin{array}{l}L_{1} \\ L_{2}\end{array}\right]=\left[\begin{array}{l}L_{1}(t, \omega) \\ L_{2}(t, \omega)\end{array}\right]$ with initial data $l \equiv\left[\begin{array}{l}L_{1} \\ L_{2}\end{array}\right] \in$ $\mathcal{L}_{\mathbb{R}}$, we can obtain solutions of this form provided that $\operatorname{Ker} L_{1}(t, \omega)$ is constant and non-zero in a half-line. This is related to the study of the properties of the so-called proper focal points, which is a modification, for the abnormal case, of the traditional notion of focal point.

A point $t_{0} \in \mathbb{R}$ is a focal point for $\left[\begin{array}{l}L_{1}(t, \omega) \\ L_{2}(t, \omega)\end{array}\right]$ if $\operatorname{det} L_{1}\left(t_{0}, \omega\right)=0$, which means that this solution intersects the Maslov cycle $\mathcal{C}$, defined in Section 2.3, at $t_{0}$. The multiplicity of a focal point is defined by $n\left(t_{0}\right)=\operatorname{dim} \operatorname{Ker} L_{1}\left(t_{0}, \omega\right)$.

Among these focal points, the so-called proper focal points (see Definition 1.1 of [33]) are fundamental in the analysis of the oscillatory properties of the Hamiltonian systems (2.1) when $H_{3}$ is positive semidefinite. It is shown in Theorem 3 of [19] that if

$$
H_{3}(\omega) \geq 0 \quad \text { for each } \omega \in \Omega,
$$

then the solution $\left[\begin{array}{l}L_{1}(t, \omega) \\ L_{2}(t, \omega)\end{array}\right] \in \mathcal{L}_{\mathbb{R}}$ is piecewise constant on any finite interval $[a, b] \subset$ $\mathbb{R}$, that is, there exists a finite number of points $a=t_{1}<t_{2}<\cdots<t_{p}=b$ such that $\operatorname{Ker} L_{1}(t, \omega)$ is constant on $\left(t_{j}, t_{j+1}\right)$ for $j=1, \ldots, p-1$ and such that

$$
\operatorname{Ker} L_{1}(t, \omega) \subseteq \operatorname{Ker} L_{1}\left(t_{j}, \omega\right) \cap \operatorname{Ker} L_{1}\left(t_{j+1}, \omega\right)
$$

for any $t \in\left(t_{j}, t_{j+1}\right)$. This result also follows easily from the proof of Lemma 3.6(a) in [6] and it justifies the equivalence stated in the next definition.

Definition 3.5. A point $t_{0} \in \mathbb{R}$ is a proper focal point for $\left[\begin{array}{l}L_{1}(t, \omega) \\ L_{2}(t, \omega)\end{array}\right]$ if

$$
\operatorname{Ker} L_{1}\left(t_{0}^{-}, \omega\right) \varsubsetneqq \operatorname{Ker} L_{1}\left(t_{0}, \omega\right),
$$

where $\operatorname{Ker} L_{1}\left(t_{0}^{-}, \omega\right)$ denotes the left-hand limit of the constant kernel of $L_{1}(t, \omega)$ at the point $t_{0}$. Or equivalently,

$$
m\left(t_{0}\right)=\operatorname{dim} \operatorname{Ker} L_{1}\left(t_{0}, \omega\right)-\operatorname{dim} \operatorname{Ker} L_{1}\left(t_{0}^{-}, \omega\right) \geq 1 .
$$

In this case, $m\left(t_{0}\right)$ is the multiplicity of the proper focal point $t_{0}$.

As a consequence, every conjoined basis has a finite number of proper focal points in each bounded subinterval of $\mathbb{R}$, although in a positive half-line it may have infinitely many proper focal points.

Let $\operatorname{Arg}$ be any argument on the set of symplectic matrices $\operatorname{Sp}(n, \mathbb{R})$ equivalent to $\operatorname{Arg}_{3} V=\arg \operatorname{det}\left(V_{1}+i V_{3}\right)$ (where $V=\left[\begin{array}{ll}V_{1} & V_{3} \\ V_{2} & V_{4}\end{array}\right]$ ). See [35] and [37] for the precise 
definitions of arguments and of equivalence between them. Given a real symplectic

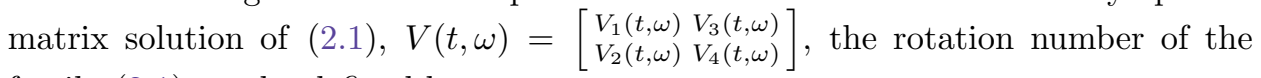
family (2.1) can be defined by

$$
\alpha=\lim _{t \rightarrow \infty} \frac{1}{t} \operatorname{Arg} V(t, \omega),
$$

where a continuous branch of the argument is taken along the curve. As shown in [23], the existence and the value of the limit (3.3) are independent of the choices of Arg and of $V(t, \omega)$. In addition, given a normalized $\sigma$-invariant measure $m_{0}$, there is a $\sigma$-invariant subset $\Omega_{0} \subseteq \Omega$ with $m_{0}\left(\Omega_{0}\right)=1$ such that the limit exists for every $\omega \in \Omega_{0}$ and takes the same constant value. We refer to this constant as the rotation number with respect to $m_{0}$.

The next result provides a new formula for the rotation number in terms of the multiplicity of the proper focal points of a conjoined basis.

Theorem 3.6. Let $\left[\begin{array}{l}L_{1}(t, \omega) \\ L_{2}(t, \omega)\end{array}\right] \in \mathcal{L}_{\mathbb{R}}$ be a matrix solution of $(2.1), \omega \in \Omega$ and denote by $J_{t}$ the set of its proper focal points contained in the interval $(0, t]$. Then the rotation number of the family (2.1) with respect to $m_{0}$ can be calculated as

$$
\alpha=\lim _{t \rightarrow \infty} \frac{\pi}{t} \sum_{t^{*} \in J_{t}} m\left(t^{*}\right) .
$$

Proof. We fix $\omega \in \Omega$, take $\left[\begin{array}{l}L_{1}(0, \omega) \\ L_{2}(0, \omega)\end{array}\right] \equiv l \in \mathcal{L}_{\mathbb{R}}$, and choose $\left[\begin{array}{l}L_{3}(0, \omega) \\ L_{4}(0, \omega)\end{array}\right] \equiv l_{1} \in \mathcal{L}_{\mathbb{R}}$ such that $\left[\begin{array}{l}L_{3}(0, \omega) L_{1}(0, \omega) \\ L_{4}(0, \omega) L_{2}(0, \omega)\end{array}\right] \in \operatorname{Sp}(n, \mathbb{R})$ (for instance, $L_{3}=L_{2} R^{-1}$ and $L_{4}=-L_{1} R^{-1}$ for $R=L_{1}^{T} L_{1}+L_{2}^{T} L_{2}$ at $\left.(0, \omega)\right)$. Then

$$
V(t, \omega)=U(t, \omega)\left[\begin{array}{ll}
L_{3}(0, \omega) & L_{1}(0, \omega) \\
L_{4}(0, \omega) & L_{2}(0, \omega)
\end{array}\right]=\left[\begin{array}{ll}
L_{3}(t, \omega) & L_{1}(t, \omega) \\
L_{4}(t, \omega) & L_{2}(t, \omega)
\end{array}\right]
$$

is a symplectic matrix solution of (2.1). We define the unitary and hence diagonalizable matrix

$$
W_{V}(t)=\left(L_{3}(t, \omega)-i L_{1}(t, \omega)\right)^{-1}\left(L_{3}(t, \omega)+i L_{1}(t, \omega)\right) .
$$

Theorem II.5.2 of [17] and Lemma 5.1 of [14] ensure the existence of continuous functions $\rho_{1}, \ldots, \rho_{n}: \mathbb{R} \rightarrow \mathbb{C}$ with $\left|\rho_{j}(t)\right|=1$ for $j=1, \ldots, n$ and $t \in \mathbb{R}$, such that the set of eigenvalues of $W_{V}(t)$, repeated according to their multiplicities, coincides with the unordered $n$-uple $\left\{\rho_{1}(t), \ldots, \rho_{n}(t)\right\}$, and 1 is an eigenvalue of $W_{V}$ if and only if $\operatorname{det} L_{1}=0$. Let $\varphi_{1}, \ldots, \varphi_{n}: \mathbb{R} \rightarrow \mathbb{R}$ be continuous argument functions: $\rho_{j}(t)=e^{i \varphi_{j}(t)}$ for $j=1, \ldots, n$ and $t \in \mathbb{R}$. Under assumption (3.2), it is known that the continuous function $\varphi_{j}: \mathbb{R} \rightarrow \mathbb{R}$ is nondecreasing for $j=1, \ldots, n$. A sketch of the proof of this result, essentially due to Lidskil [20], is given in Proposition 2.4 of [4].

As a consequence of all this, $\varphi(t)=(1 / 2) \sum_{j=1}^{n} \varphi_{j}(t)$ is a continuous branch of $\operatorname{Arg}_{3} V(t, \omega)=\arg \operatorname{det}\left(L_{3}(t, \omega)+i L_{1}(t, \omega)\right)$, which implies that

$$
\alpha=\lim _{t \rightarrow \infty} \frac{1}{t} \operatorname{Arg}_{3} V(t, \omega)=\lim _{t \rightarrow \infty} \frac{1}{t} \varphi(t),
$$

and the statement of the theorem holds if we check that

$$
\sum_{j=1}^{n} \varphi_{j}(t)=\sum_{j=1}^{n} \varphi_{j}(0)+2 \pi \sum_{t^{*} \in J_{t}} m\left(t^{*}\right)+\widetilde{r}_{t}
$$


with $\left|\widetilde{r}_{t}\right| \leq 2 \pi n$ for all $t \in(0, \infty)$.

Let $t_{0}$ be the first proper focal point in $(0, \infty)$. As stated above, det $L_{1}\left(t_{0}, \omega\right)=0$ if and only if 1 is an eigenvalue of $W_{V}\left(t_{0}\right)$ or equivalently, there is a $j$ such that $\varphi_{j}\left(t_{0}\right) \equiv 0 \bmod 2 \pi$. Moreover, $\operatorname{dim} \operatorname{Ker} L_{1}\left(t_{0}, \omega\right)=\operatorname{dim} \operatorname{Ker} L_{1}\left(t_{0}^{-}, \omega\right)+m\left(t_{0}\right)$ and consequently, since $W_{V}(t)$ is diagonalizable and the multiplicity of its eigenvalue 1 coincide with $\operatorname{dim} \operatorname{Ker} L_{1}(t, \omega), m\left(t_{0}\right)$ measures the increment of the number of eigenvalues satisfying $\varphi_{j}\left(t_{0}\right) \equiv 0 \bmod 2 \pi$ from the left. Hence, from the nondecreasing character of the arguments, since there are exactly $m\left(t_{0}\right)$ arguments for which $\left|\varphi_{j}\left(t_{0}\right)-\varphi_{j}(0)-2 \pi\right| \leq 2 \pi$, and the variation of the rest of argument lies in an interval of length $2 \pi$, the total sum satisfies

$$
\sum_{j=1}^{n} \varphi_{j}\left(t_{0}\right)=\sum_{j=1}^{n} \varphi_{j}(0)+2 \pi m\left(t_{0}\right)+\widetilde{r}_{t_{0}}
$$

for some $\left|\widetilde{r}_{t_{0}}\right| \leq 2 \pi n$. This equality is also valid substituting $t_{0}$ by any $t \in\left[t_{0}, t_{1}\right)$, where $t_{1}$ denotes the next proper focal point. Analogously, the number of times that the argument $0 \bmod 2 \pi$ has been strictly reached (not being constant from the left) in the interval $\left(0, t_{2}\right)$ ( $t_{2}$ being the next proper focal point) is $m\left(t_{0}\right)+m\left(t_{1}\right)$, and we obtain

$$
\sum_{j=1}^{n} \varphi_{j}(t)=\sum_{j=1}^{n} \varphi_{j}(0)+2 \pi m\left(t_{0}\right)+2 \pi m\left(t_{1}\right)+\widetilde{r}_{t}
$$

for $t \in\left[t_{1}, t_{2}\right)$ and some $\left|\widetilde{r}_{t}\right| \leq 2 \pi n$. Completely similar arguments prove the formula (3.4) and the proof is finished. Notice that if there are no proper focal points in $(0, \infty)$, then it is immediate to check that $\alpha=0$.

This theorem gives precise significance to the interpretation of the rotation number as "the average number of times a given solution curve intersects the Maslov cycle". Also it is worth noting that $2 \pi n$ is not the optimal bound for $\widetilde{r}_{t}$ when $t>t_{0}$.

Recall that the linear Hamiltonian system (2.1) is said to be nonoscillatory at $+\infty$ if $\operatorname{Arg} V(t, \omega)$ is a bounded function on $[a, \infty)$, where $V(t, \omega)$ is any symplectic fundamental matrix solution and a continuous branch of the argument is taken along the curve. Under condition (3.2), the previous result provides an equivalent characterization of a linear Hamiltonian system (2.1) as nonoscillatory (resp. oscillatory) at $+\infty$ if each solution $\left[\begin{array}{l}L_{1}(t, \omega) \\ L_{2}(t, \omega)\end{array}\right] \in \mathcal{L}_{\mathbb{R}}$ has a finite number (resp. infinite number) of proper focal points in $[a, \infty)$, a definition which has already been introduced in the literature (see for instance [29]).

In addition, as we have stated above, under assumption (3.2) each solution $\left[\begin{array}{l}L_{1}(t, \omega) \\ L_{2}(t, \omega)\end{array}\right] \in \mathcal{L}_{\mathbb{R}}$ with initial data $l \in \mathcal{L}_{\mathbb{R}}$ has $\operatorname{Ker} L_{1}(t, \omega)$ piecewise constant on any compact subinterval of $[a, \infty)$, and then, in the nonoscillatory case there is a $t_{0}^{+}(\omega)\left(\right.$ resp. $\left.t_{0}^{-}(\omega)\right)$ such that $\operatorname{Ker} L_{1}(t, \omega)$ is constant for each $t \geq t_{0}^{+}(\omega)$ (resp. $\left.t \leq t_{0}^{-}(\omega)\right)$. As a consequence, in the nonoscillatory case at $+\infty$ (resp. at $-\infty$ )

$$
d^{+}(\omega, l)=\operatorname{dim} \operatorname{Ker} L_{1}\left(t_{0}^{+}(\omega), \omega\right) \quad\left(\text { resp. } d^{-}(\omega, l)=\operatorname{dim} \operatorname{Ker} L_{1}\left(t_{0}^{-}(\omega), \omega\right)\right) .
$$

\section{Linear-QUADRATIC Dissipative CONTROL SYSTEMS}

We next study and adapt the concept of dissipative control system introduced by Willems (see $[34,32]$ ) to the case of a time-varying linear control system with a linear-quadratic supply rate. 
Consider the control system

$$
\mathbf{x}^{\prime}=A(t) \mathbf{x}+B(t) \mathbf{u},
$$

where $\mathbf{x} \in \mathbb{R}^{n}$ and $\mathbf{u} \in \mathbb{R}^{m}$, together with the quadratic form

$$
\mathcal{Q}(t, \mathbf{x}, \mathbf{u})=\frac{1}{2}(\langle\mathbf{x}, G(t) \mathbf{x}\rangle+2\langle\mathbf{x}, g(t) \mathbf{u}\rangle+\langle\mathbf{u}, R(t) \mathbf{u}\rangle) .
$$

The functions $A, B, G, g$ and $R$ are assumed to be bounded and uniformly continuous functions on $\mathbb{R}$, with values in the sets of real matrices of the appropriate dimensions. In addition, $G$ and $R$ are symmetric, and $R \geq \delta I_{m}$ for a $\delta>0$.

The notion of dissipativity is related to that of a storage function.

Definition 4.1. A function $V: \mathbb{R} \times \mathbb{R}^{n} \rightarrow \mathbb{R}$ is a storage function for the control system (4.1) with supply rate $\mathcal{Q}$ if the following conditions hold. First, $V(t, 0)=0$ and $V(t, \mathbf{x}) \geq 0$ for all $(t, \mathbf{x}) \in \mathbb{R} \times \mathbb{R}^{n}$. Second, if $t_{1}<t_{2}$, if $\mathbf{u}:\left[t_{1}, t_{2}\right] \rightarrow \mathbb{R}^{m}$ is a square integrable control function, and if $\mathbf{x}(t)$ solves the corresponding system (4.1) (with arbitrary initial value $\mathbf{x}\left(t_{1}\right) \in \mathbb{R}^{n}$ ), then

$$
\int_{t_{1}}^{t_{2}} \mathcal{Q}(t, \mathbf{x}(t), \mathbf{u}(t)) d t \geq V\left(t_{2}, \mathbf{x}\left(t_{2}\right)\right)-V\left(t_{1}, \mathbf{x}\left(t_{1}\right)\right) .
$$

The function $V$ is a strong storage function for (4.1) with supply rate $\mathcal{Q}$ if it is a storage function and if, in addition, $V(t, \mathbf{x})>0$ for all $t \in \mathbb{R}$ and all nonzero $\mathbf{x} \in \mathbb{R}^{n}$.

Definition 4.2. The control system (4.1) is dissipative with supply rate $\mathcal{Q}$ if there is a storage function $V$ with supply rate $\mathcal{Q}$. The control system (4.1) is strictly dissipative with supply rate $\mathcal{Q}$ if there exists $\delta>0$ such that (4.1) is dissipative with the modified supply rate

$$
\mathcal{Q}^{\delta}(t, \mathbf{x}, \mathbf{u})=\mathcal{Q}(t, \mathbf{x}, \mathbf{u})-\delta\left(\|\mathbf{x}\|^{2}+\|\mathbf{u}\|^{2}\right) .
$$

Generally speaking, our system exchanges energy with its environment, and this phenomenon is modelled by the supply rate $\mathcal{Q}$ : when this quantity is integrated it measures the flow of energy from the environment into the system. The storage function measures the quantity of energy stored inside the system. The inequality (4.3) formalizes the idea that a dissipative system is characterized by the property that the change of internal stored energy in a given time interval will never exceed the amount of energy that flows into the system in that interval.

A dissipative system is characterized by the existence of the available storage next defined. As shown later, it turns out to be the minimum of all the storage functions for the control system.

Definition 4.3. The available storage of the control system (4.1) for $\mathcal{Q}$ is the extended-real function $V_{a}$ defined on $\mathbb{R} \times \mathbb{R}^{n}$ by

$$
V_{a}(t, \mathbf{x})=\sup _{h \geq 0}\left\{\begin{array}{l|l}
-\int_{t}^{t+h} \mathcal{Q}(s, \mathbf{x}(s), \mathbf{u}(s)) d s & \begin{array}{l}
\mathbf{u}:[t, t+h] \rightarrow \mathbb{R}^{m} \text { control and } \\
\mathbf{x} \text { solution of }(4.1) \text { with } \mathbf{x}(t)=\mathbf{x}
\end{array}
\end{array}\right\} .
$$

Notice that $V_{a}(t, \mathbf{x}) \geq 0$. The next result is a nonautonomous version of Theorem 1 of [34].

Proposition 4.4. The following statements are equivalent:

(i) The control system (4.1) is dissipative with supply rate $\mathcal{Q}$. 
(ii) The available storage $V_{a}$ for $\mathcal{Q}$ satisfies $V_{a}(t, \mathbf{x})<\infty$ for each $(t, \mathbf{x}) \in$ $\mathbb{R} \times \mathbb{R}^{n}$.

Under these conditions $V_{a}$ is a storage function for (4.1) with supply rate $\mathcal{Q}$ and $V_{a} \leq V$ for any other storage function $V$.

Proof. (i) $\Rightarrow$ (ii) Let $V$ be a storage function for (4.1) with supply rate $\mathcal{Q}$. Since $V$ is nonnegative

$$
-\int_{t}^{t+h} \mathcal{Q}(s, \mathbf{x}(s), \mathbf{u}(s)) d s \leq V(t, \mathbf{x}(t))-V(t+h, \mathbf{x}(t+h)) \leq V(t, \mathbf{x}(t))<\infty,
$$

so that $V_{a}(t, \mathbf{x})<\infty$ and $V_{a}(t, \mathbf{x}) \leq V(t, \mathbf{x})$ for each $(t, \mathbf{x}) \in \mathbb{R} \times \mathbb{R}^{n}$.

(ii) $\Rightarrow$ (i) Assume that $V_{a}(t, \mathbf{x})<\infty$ for each $(t, \mathbf{x}) \in \mathbb{R} \times \mathbb{R}^{n}$. We consider a square integrable control function $\mathbf{u}:\left[t_{1}, t_{2}\right] \rightarrow \mathbb{R}^{m}$ and a solution $\mathbf{x}:\left[t_{1}, t_{2}\right] \rightarrow$ $\mathbb{R}^{n}$ of (4.1). For any $h \geq 0$, we take any control $\widetilde{\mathbf{u}}:\left[t_{2}, t_{2}+h\right] \rightarrow \mathbb{R}^{m}$ and the solution $\widetilde{\mathbf{x}}:\left[t_{2}, t_{2}+h\right] \rightarrow \mathbb{R}^{n}$ of (4.1) for $\widetilde{\mathbf{u}}$ with $\widetilde{\mathbf{x}}\left(t_{2}\right)=\mathbf{x}\left(t_{2}\right)$. We denote by $\widehat{\mathbf{x}}:\left[t_{1}, t_{2}+h\right] \rightarrow \mathbb{R}^{n}$ and $\widehat{\mathbf{u}}:\left[t_{1}, t_{2}+h\right] \rightarrow \mathbb{R}^{m}$ the concatenations of $\mathbf{x}$ with $\widetilde{\mathbf{x}}$ and $\mathbf{u}$ with $\widetilde{\mathbf{u}}$ respectively. Note that $\widehat{\mathbf{x}}$ coincides with the solution of (4.1) for $\widehat{\mathbf{u}}$ with $\widehat{\mathbf{x}}\left(t_{1}\right)=\mathbf{x}\left(t_{1}\right)$. From the definition of $V_{a}$

$$
\begin{aligned}
V_{a}\left(t_{1}, \mathbf{x}\left(t_{1}\right)\right) & \geq-\int_{t_{1}}^{t_{2}+h} \mathcal{Q}(s, \widehat{\mathbf{x}}(s), \widehat{\mathbf{u}}(s)) d s \\
& =-\int_{t_{1}}^{t_{2}} \mathcal{Q}(s, \mathbf{x}(s), \mathbf{u}(s)) d s-\int_{t_{2}}^{t_{2}+h} \mathcal{Q}(s, \widetilde{\mathbf{x}}(s), \widetilde{\mathbf{u}}(s)) d s .
\end{aligned}
$$

Taking the supremum on $h \geq 0$ and $\widetilde{\mathbf{u}}$ we conclude that

$$
V_{a}\left(t_{1}, \mathbf{x}\left(t_{1}\right)\right) \geq-\int_{t_{1}}^{t_{2}} \mathcal{Q}(s, \mathbf{x}(s), \mathbf{u}(s)) d s+V_{a}\left(t_{2}, \mathbf{x}\left(t_{2}\right)\right)
$$

that is,

$$
\int_{t_{1}}^{t_{2}} \mathcal{Q}(s, \mathbf{x}(s), \mathbf{u}(s)) d s \geq V_{a}\left(t_{2}, \mathbf{x}\left(t_{2}\right)\right)-V_{a}\left(t_{1}, \mathbf{x}\left(t_{1}\right)\right) .
$$

In order to check that $V_{a}$ is a storage function with supply rate $\mathcal{Q}$, it remains to prove that $V_{a}(t, 0)=0$ for each $t \in \mathbb{R}$. Given $\varepsilon>0$ there exist $h_{\varepsilon} \geq 0$, a control $\mathbf{u}_{\varepsilon}:\left[t, t+h_{\varepsilon}\right] \rightarrow \mathbb{R}^{m}$ and a solution $\mathbf{x}_{\varepsilon}:\left[t, t+h_{\varepsilon}\right] \rightarrow \mathbb{R}^{n}$ of $(4.1)$ with $\mathbf{x}_{\varepsilon}(t)=0$ such that

$$
V_{a}(t, 0) \leq-\int_{t}^{t+h_{\varepsilon}} \mathcal{Q}\left(s, \mathbf{x}_{\varepsilon}(s), \mathbf{u}_{\varepsilon}(s)\right) d s+\varepsilon
$$

Since $\mathcal{Q}$ is a quadratic form, for each $\lambda>0$

$$
V_{a}(t, 0) \leq-\frac{1}{\lambda^{2}} \int_{t}^{t+h_{\varepsilon}} \mathcal{Q}\left(s, \lambda \mathbf{x}_{\varepsilon}(s), \lambda \mathbf{u}_{\varepsilon}(s)\right) d s+\varepsilon \leq \frac{1}{\lambda^{2}} V_{a}(t, 0)+\varepsilon,
$$

and taking the limit as $\lambda \uparrow \infty$, we deduce that $V_{a}(t, 0) \leq \varepsilon$ for each $\varepsilon>0$, that is, $V_{a}(t, 0)=0$, which finishes the proof.

The analysis which follows will be carried out for a family of control systems defined over a compact metric space $\Omega$ with a continuous flow $\sigma$. In particular, in the case in which $\Omega$ is constructed as the hull of the initial data $(A, B, G, g, R)$ in a suitable topology, the results regarding the dissipativity of the initial system (4.1) can be derived from the results concerning the family (4.4) below by an obvious 
"restriction" process. See also [6, 5] and [15] regarding the advantages of a "collective" study.

So, let $A, G: \Omega \rightarrow \mathbb{M}_{n \times n}(\mathbb{R}), B, g: \Omega \rightarrow \mathbb{M}_{n \times m}(\mathbb{R})$ and $R: \Omega \rightarrow \mathbb{M}_{m \times m}(\mathbb{R})$ be continuous matrix-valued functions, with $G$ and $R$ symmetric and $R>0$. We consider the family of control systems

$$
\mathbf{x}^{\prime}=A(\omega \cdot t) \mathbf{x}+B(\omega \cdot t) \mathbf{u},
$$

together with the family of quadratic functionals

$$
\mathcal{Q}_{\omega}(t, \mathbf{x}, \mathbf{u})=\frac{1}{2}(\langle\mathbf{x}, G(\omega \cdot t) \mathbf{x}\rangle+2\langle\mathbf{x}, g(\omega \cdot t) \mathbf{u}\rangle+\langle\mathbf{u}, R(\omega \cdot t) \mathbf{u}\rangle),
$$

for $\omega \in \Omega$, as well as the family of time-reversed control systems

$$
\mathbf{x}^{\prime}=-A(\omega \cdot(-t)) \mathbf{x}-B(\omega \cdot(-t)) \mathbf{u},
$$

together with the family of time-reversed quadratic functionals

$$
\mathcal{Q}_{\omega}^{-}(t, \mathbf{x}, \mathbf{u})=\frac{1}{2}(\langle\mathbf{x}, G(\omega \cdot(-t)) \mathbf{x}\rangle+2\langle\mathbf{x}, g(\omega \cdot(-t)) \mathbf{u}\rangle+\langle\mathbf{u}, R(\omega \cdot(-t)) \mathbf{u}\rangle)
$$

Definition 4.5. The system (4.4) is null controllable if for each $\mathbf{x}_{0} \in \mathbb{R}^{n}$ there exist a time $t_{0}>0$ and an integrable control function $\mathbf{u}:\left[0, t_{0}\right] \rightarrow \mathbb{R}^{m}$ such that the solution $\mathbf{x}(t)$ with $\mathbf{x}(0)=\mathbf{x}_{0}$ satisfies $\mathbf{x}\left(t_{0}\right)=0$. In this case, the control $\mathbf{u}$ steers $\mathbf{x}_{0}$ to 0 in time $t_{0}$.

Definition 4.6. The family (4.4) is uniformly null controllable if all the systems of the family are null controllable with a common time $t_{0}>0$.

We refer the reader to Johnson and Nerurkar [12] for the proofs of the following remarks.

Remarks 4.7. 1. The definition of uniform null controllability is equivalent to the existence of numbers $t_{0}>0$ and $\delta>0$ such that for all $\omega \in \Omega$,

$$
\int_{0}^{t_{0}} U_{A}^{-1}(t, \omega) B(\omega \cdot t) B^{T}(\omega \cdot t)\left(U_{A}^{-1}\right)^{T}(t, \omega) d t \geq \delta I_{n}
$$

where $U_{A}(t, \omega)$ is the fundamental matrix solution of the system $\mathbf{x}^{\prime}=A(\omega \cdot t) \mathbf{x}$ with $U_{A}(0, \omega)=I_{n}$.

2. The family (4.4) is uniformly null controllable if and only if each minimal subset of $\Omega$ contains at least one point $\omega_{1}$ such that the corresponding system (4.4) for $\omega_{1}$ is null controllable.

The next result is proved in Proposition 2.5 of [5].

Proposition 4.8. If the family of control systems (4.4) is uniformly null controllable so is the family of time-reversed control systems (4.6).

The next result provides a characterization of the dissipativity of the family of control systems (4.4) under the hypothesis of uniform null controllability.

Proposition 4.9. Assume that the family (4.4) is uniformly null controllable. Fix $\omega \in \Omega$. Then the following statements are equivalent:

(i) The control system (4.4) is dissipative with supply rate $\mathcal{Q}_{\omega}$. 
(ii) For each pair $t_{1}<t_{2}$ and for each square integrable control $\mathbf{u}:\left[t_{1}, t_{2}\right] \rightarrow \mathbb{R}^{m}$, the solution $\mathbf{x}(t)$ of (4.4) satisfying $\mathbf{x}\left(t_{1}\right)=0$ has the property that

$$
\int_{t_{1}}^{t_{2}} \mathcal{Q}_{\omega}(s, \mathbf{x}(s), \mathbf{u}(s)) d s \geq 0 .
$$

Proof. (i) $\Rightarrow$ (ii) Let $V^{\omega}$ be a storage function for (4.4) with supply rate $\mathcal{Q}_{\omega}$. Then, for a pair $(\mathbf{x}, \mathbf{u})$ as in (ii),

$$
\int_{t_{1}}^{t_{2}} \mathcal{Q}_{\omega}(s, \mathbf{x}(s), \mathbf{u}(s)) d s \geq V^{\omega}\left(t_{2}, \mathbf{x}\left(t_{2}\right)\right)-V^{\omega}\left(t_{1}, \mathbf{x}\left(t_{1}\right)\right)=V^{\omega}\left(t_{2}, \mathbf{x}\left(t_{2}\right)\right) \geq 0,
$$

and (ii) is satisfied.

(ii) $\Rightarrow$ (i) From Proposition 4.4 it is enough to check that the available storage of the control system (4.4)

$$
V_{a}^{\omega}(t, \mathbf{x})=\sup _{h \geq 0}\left\{\begin{array}{l|l}
-\int_{t}^{t+h} \mathcal{Q}_{\omega}(s, \mathbf{x}(s), \mathbf{u}(s)) d s & \begin{array}{l}
\mathbf{u}:[t, t+h] \rightarrow \mathbb{R}^{m} \text { control and } \\
\mathbf{x} \text { solution of }(4.4) \text { with } \mathbf{x}(t)=\mathbf{x}
\end{array}
\end{array}\right\}
$$

satisfies $V_{a}^{\omega}(t, \mathbf{x})<\infty$.

Fix $(t, \mathbf{x}) \in \mathbb{R} \times \mathbb{R}^{n}$. Proposition 4.8 shows that the time-reversed problem (4.6) is uniformly null controllable. It is easy to deduce that there exist a $t_{1}<t$ and a control $\widehat{\mathbf{u}}:\left[t_{1}, t\right] \rightarrow \mathbb{R}^{n}$ such that the solution $\widehat{\mathbf{x}}:\left[t_{1}, t\right] \rightarrow \mathbb{R}^{n}$ for (4.4) for the control $\widehat{\mathbf{u}}$ and with $\widehat{\mathbf{x}}(t)=\mathbf{x}$ satisfies $\widehat{\mathbf{x}}\left(t_{1}\right)=0$. Now, given any control $\mathbf{u}:[t, t+h] \rightarrow \mathbb{R}^{m}$, we define $\mathbf{u}^{*}:\left[t_{1}, t+h\right] \rightarrow \mathbb{R}^{m}$ concatenating $\widehat{\mathbf{u}}$ with $\mathbf{u}$ and denote by $\mathbf{x}^{*}$ the solution of (4.4) for this control with $\mathbf{x}^{*}\left(t_{1}\right)=0$. Note that $\mathbf{x}^{*}$ agrees with $\widehat{\mathbf{x}}$ in $\left[t_{1}, t\right]$, and with the solution $\mathbf{x}$ of (4.4) satisfying $\mathbf{x}(t)=\mathbf{x}$ in $[t, t+h]$. In addition, the assumption in (ii) ensures that

$$
\int_{t_{1}}^{t+h} \mathcal{Q}_{\omega}\left(s, \mathbf{x}^{*}(s), \mathbf{u}^{*}(s)\right) d s \geq 0
$$

that is,

and hence,

$$
\int_{t_{1}}^{t} \mathcal{Q}_{\omega}(s, \widehat{\mathbf{x}}(s), \widehat{\mathbf{u}}(s)) d s \geq-\int_{t}^{t+h} \mathcal{Q}_{\omega}(s, \mathbf{x}(s), \mathbf{u}(s)) d s,
$$

as stated.

$$
V_{a}^{\omega}(t, \mathbf{x}) \leq \int_{t_{1}}^{t} \mathcal{Q}_{\omega}(s, \widehat{\mathbf{x}}(s), \widehat{\mathbf{u}}(s)) d s<\infty,
$$

Proposition 4.10. Assume that the family (4.4) is uniformly null controllable and let $\omega_{0} \in \Omega$ be such that the control system of the family (4.4) for $\omega=\omega_{0}$ is dissipative with supply rate $\mathcal{Q}_{\omega_{0}}$. Then for each $\omega_{1} \in \operatorname{cls}\left\{\omega_{0} \cdot t \mid t \in \mathbb{R}\right\}$ the control system of (4.4) for $\omega=\omega_{1}$ is dissipative with supply rate $\mathcal{Q}_{\omega_{1}}$.

Proof. Let $\mathbf{u}:\left[t_{1}, t_{2}\right] \rightarrow \mathbb{R}^{m}$ be a square integrable control and let $\mathbf{x}(t)$ be the solution of (4.4) for $\mathbf{u}$ and $\omega=\omega_{1}$ with $\mathbf{x}\left(t_{1}\right)=0$. From Proposition 4.9 it is enough to check that

$$
\int_{t_{1}}^{t_{2}} \mathcal{Q}_{\omega_{1}}(s, \mathbf{x}(s), \mathbf{u}(s)) d s \geq 0 .
$$

Since $\omega_{1} \in \operatorname{cls}\left\{\omega_{0} \cdot t \mid t \in \mathbb{R}\right\}$, we can take a sequence $\left(t_{n}\right)$ with $\lim _{n \rightarrow \infty} \omega_{0} \cdot t_{n}=\omega_{1}$. Then

$$
\int_{t_{1}}^{t_{2}} \mathcal{Q}_{\omega_{1}}(s, \mathbf{x}(s), \mathbf{u}(s)) d s=\lim _{n \rightarrow \infty} \int_{t_{1}}^{t_{2}} \mathcal{Q}_{\omega_{0} \cdot t_{n}}\left(s, \mathbf{x}_{n}(s), \mathbf{u}(s)\right) d s
$$


where $\mathbf{x}_{n}$ is the solution of (4.4) for $\mathbf{u}$ and $\omega=\omega_{0} \cdot t_{n}$ with $\mathbf{x}_{n}\left(t_{1}\right)=0$. Moreover, from (4.5),

$$
\mathcal{Q}_{\omega_{0} \cdot t_{n}}\left(s, \mathbf{x}_{n}(s), \mathbf{u}(s)\right) d s=\mathcal{Q}_{\omega_{0}}\left(s+t_{n}, \mathbf{x}_{n}(s), \mathbf{u}(s)\right),
$$

and since for $\omega_{0}$ the control system (4.4) is dissipative with supply rate $\mathcal{Q}_{\omega_{0}}$, again from Proposition 4.9 we deduce that

$$
\int_{t_{1}}^{t_{2}} \mathcal{Q}_{\omega_{1}}(s, \mathbf{x}(s), \mathbf{u}(s)) d s=\lim _{n \rightarrow \infty} \int_{t_{1}+t_{n}}^{t_{2}+t_{n}} \mathcal{Q}_{\omega_{0}}\left(s, \mathbf{x}_{n}\left(s-t_{n}\right), \mathbf{u}\left(s-t_{n}\right)\right) d s \geq 0,
$$

as stated.

It is not hard to check that the conclusions of Propositions 4.9 and 4.10 do not hold if the uniform null controllability is not assumed.

Definition 4.11. Assume that the family (4.4) is uniformly null controllable. The required supply of the control system (4.4) for $\mathcal{Q}_{\omega}$ is the extended-real function $V_{r}^{\omega}$ defined on $\mathbb{R} \times \mathbb{R}^{n}$ by

$V_{r}^{\omega}(t, \mathbf{x})=\inf _{h \geq 0}\left\{\begin{array}{l|l}\int_{t-h}^{t} \mathcal{Q}_{\omega}(s, \mathbf{x}(s), \mathbf{u}(s)) d s & \begin{array}{l}\mathbf{u}:[t-h, t] \rightarrow \mathbb{R}^{m} \text { control and } \mathbf{x} \\ \text { satisfies }(4.4), \mathbf{x}(t)=\mathbf{x}, \mathbf{x}(t-h)=0\end{array}\end{array}\right\}$.

It is easy to deduce from Proposition 4.8 that the set in which the infimum is taken is nonempty for all $(t, \mathbf{x}) \in \mathbb{R} \times \mathbb{R}^{n}$. The next result provides a nonautonomous version of Theorem 2 of [34].

Proposition 4.12. Assume that the family (4.4) is uniformly null controllable. Fix $\omega \in \Omega$. Then the following statements are equivalent:

(i) The control system (4.4) is dissipative with supply rate $\mathcal{Q}_{\omega}$.

(ii) The required supply $V_{r}^{\omega}$ satisfies $V_{r}^{\omega}(t, \mathbf{x}) \geq 0$ for each $(t, \mathbf{x}) \in \mathbb{R} \times \mathbb{R}^{n}$.

Under these conditions, $V_{r}^{\omega}$ is a storage function for (4.4) with supply rate $\mathcal{Q}_{\omega}$ and $V^{\omega} \leq V_{r}^{\omega}$ for any other storage function $V^{\omega}$.

Proof. The equivalence of (i) and (ii) is an easy consequence of Proposition 4.9. Next we check that $V_{r}^{\omega}$ is a storage function for (4.4) with supply rate $\mathcal{Q}_{\omega}$.

Since we are assuming that $V_{r}^{\omega}(t, \mathbf{x}) \geq 0$, from the definition it is immediate to check that $V_{r}^{\omega}(t, 0)=0$. Next, take $t_{1}<t_{2}, \mathbf{u}:\left[t_{1}, t_{2}\right] \rightarrow \mathbb{R}^{m}$ a square integrable control function and $\mathbf{x}(t)$ a solution of (4.4). Again, from the definition of $V_{r}^{\omega}$, for any control $\widetilde{\mathbf{u}}:\left[t_{1}-h, t_{1}\right] \rightarrow \mathbb{R}^{m}$ and solution $\widetilde{\mathbf{x}}$ with $\widetilde{\mathbf{x}}\left(t_{1}\right)=\mathbf{x}\left(t_{1}\right)$ and $\widetilde{\mathbf{x}}\left(t_{1}-h\right)=0$ we have

$$
V_{r}^{\omega}\left(t_{2}, \mathbf{x}\left(t_{2}\right)\right) \leq \int_{t_{1}-h}^{t_{1}} \mathcal{Q}_{\omega}(s, \widetilde{\mathbf{x}}(s), \widetilde{\mathbf{u}}(s)) d s+\int_{t_{1}}^{t_{2}} \mathcal{Q}_{\omega}(s, \mathbf{x}(s), \mathbf{u}(s)) d s,
$$

and taking the infimum we deduce that

$$
V_{r}^{\omega}\left(t_{2}, \mathbf{x}\left(t_{2}\right)\right) \leq V_{r}^{\omega}\left(t_{1}, \mathbf{x}\left(t_{1}\right)\right)+\int_{t_{1}}^{t_{2}} \mathcal{Q}_{\omega}(s, \mathbf{x}(s), \mathbf{u}(s)) d s
$$

that is, $V_{r}^{\omega}$ is a storage function for (4.4) with supply rate $\mathcal{Q}_{\omega}$. Finally, for any other storage function $V^{\omega}$ for (4.4) with supply rate $\mathcal{Q}_{\omega}$, and for any control $\mathbf{u}:[t-h, t] \rightarrow$ $\mathbb{R}^{m}$ with solution $\mathbf{x}:[t-h, t] \rightarrow \mathbb{R}^{n}$ of (4.4) satisfying $\mathbf{x}(t-h)=0$ and $\mathbf{x}(t)=\mathbf{x}$, we deduce that

$$
\int_{t-h}^{t} \mathcal{Q}_{\omega}(s, \mathbf{x}(s), \mathbf{u}(s)) d s \geq V^{\omega}(t, \mathbf{x}(t))-V^{\omega}(t-h, \mathbf{x}(t-h))=V^{\omega}(t, \mathbf{x}),
$$

and taking the infimum we conclude that $V_{r}^{\omega}(t, \mathbf{x}) \geq V^{\omega}(t, \mathbf{x})$, as stated. 
4.1. Conditions for dissipativity without null controllability. Under the assumptions of uniform null controllability for the family (4.4) and the existence of exponential dichotomy for the family (4.8), necessary and sufficient conditions for its dissipative and strictly dissipative character with supply rate $\mathcal{Q}_{\omega}$ were proved in [5] and [15]. In this subsection we will obtain some characterizations omitting the condition of uniform null controllability.

As is discussed in [5] and [15], an appropriate application of the Pontryagin Maximum Principle relates (4.4) and (4.5) with the dynamical properties of the nonautonomous family of linear Hamiltonian systems

$$
\mathbf{z}^{\prime}=H(\omega \cdot t) \mathbf{z}
$$

for $\omega \in \Omega$, where $\mathbf{z}=\left[\begin{array}{l}\mathbf{x} \\ \mathbf{y}\end{array}\right]$ for $\mathbf{x}, \mathbf{y} \in \mathbb{R}^{n}$ and

$$
H(\omega)=\left[\begin{array}{cc}
A(\omega)-B(\omega) R^{-1}(\omega) g^{T}(\omega) & B(\omega) R^{-1}(\omega) B^{T}(\omega) \\
G(\omega)-g(\omega) R^{-1}(\omega) g^{T}(\omega) & -A^{T}(\omega)+g(\omega) R^{-1}(\omega) B^{T}(\omega)
\end{array}\right] .
$$

Analogously, the time-reversed control systems (4.6) and (4.7) lead to the family

$$
\mathbf{z}^{\prime}=H^{-}(\omega \cdot(-t)) \mathbf{z}
$$

for $\omega \in \Omega$, where

$$
H^{-}(\omega)=\left[\begin{array}{cc}
-A(\omega)+B(\omega) R^{-1}(\omega) g^{T}(\omega) & B(\omega) R^{-1}(\omega) B^{T}(\omega) \\
G(\omega)-g(\omega) R^{-1}(\omega) g^{T}(\omega) & A^{T}(\omega)-g(\omega) R^{-1}(\omega) B^{T}(\omega)
\end{array}\right] .
$$

Note that no additional condition is needed to construct these families from the linear-quadratic control problems. We include a consequence of Lemma 3.4 of [6] for completeness.

Lemma 4.13. The system (4.4) is null controllable if and only if

$$
\mathbf{x}^{\prime}=\widetilde{A}(\omega \cdot t) \mathbf{x}+\widetilde{B}(\omega \cdot t) \mathbf{u}
$$

is null controllable, where $\widetilde{A}=A-B R^{-1} g^{T}$ and $\widetilde{B}=B R^{-1} B^{T}$.

Remark 4.14. It follows easily from Remark 4.7.1 (see Proposition 5.1 of [14]) that the family (4.10) is uniformly null controllable if and only if none of the systems of the family (4.8) admits a nonzero solution of the form $\mathbf{z}(t, \omega)=\left[\begin{array}{c}0 \\ \mathbf{z}_{2}(t, \omega)\end{array}\right]$ on $[0, \infty)$, i.e., if and only if $d^{+}(\omega)=0$ for all $\omega \in \Omega$. Consequently, Remark 4.7.2 and Lemma 4.13 allow us to assert that the family (4.4) is uniformly null controllable if and only if each minimal subset of $\Omega$ contains at least one point $\omega_{1}$ such that (4.8) satisfies $d^{+}\left(\omega_{1}\right)=0$.

As explained in Subsection 2.3, $M\left(t, \omega_{0}, M_{0}\right)$ denotes the solution of the corresponding Riccati equation (2.5) associated to (4.8), that is

$$
M^{\prime}=-M H_{3}(\omega \cdot t) M-M H_{1}(\omega \cdot t)-H_{1}^{T}(\omega \cdot t) M+H_{2}(\omega \cdot t)
$$

with $H_{1}=A-B R^{-1} g^{T}, H_{2}=G-g R^{-1} g^{T}$ and $H_{3}=B R^{-1} B^{T}$, for $\omega=\omega_{0}$ and $M\left(0, \omega_{0}, M_{0}\right)=M_{0}$.

From Remark 4.14, when the family of control systems (4.4) is not uniformly null controllable, there are minimal subsets of $\Omega$ for which all the systems are abnormal at $+\infty$, and consequently, abnormal (see Theorem 3.1). However, it is possible to have the coexistence of points $\omega \in \Omega$ for which the corresponding control system (4.4) is dissipative with others for which this property does not hold, even 
in the same minimal subset. The next result provide conditions for the dissipativity of one particular system of the family. Note that the exponential dichotomy of the family (4.8) is not assumed in this case.

Proposition 4.15. $\quad$ (i) Assume that there is an $\omega_{0} \in \Omega$ and a positive semidefinite matrix $M_{0} \geq 0$ such that $M\left(t, \omega_{0}, M_{0}\right)$ is globally defined and positive semidefinite for all $t \in \mathbb{R}$. Then the system (4.4) for $\omega_{0}$ is dissipative with supply rate $\mathcal{Q}_{\omega_{0}}$.

(ii) Assume that there is a $\omega_{0} \in \Omega$ with dense orbit, i.e., $\operatorname{cls}\left\{\omega_{0} \cdot t \mid t \in \mathbb{R}\right\}=$ $\Omega$, and a positive semidefinite matrix $M_{0} \geq 0$ such that $M\left(t, \omega_{0}, M_{0}\right)$ is globally defined, bounded and positive semidefinite for all $t \in \mathbb{R}$. Then the system (4.4) is dissipative with supply rate $\mathcal{Q}_{\omega}$ for each $\omega \in \Omega$.

Proof. (i) We define

$$
V^{\omega_{0}}(t, \mathbf{x})=\frac{1}{2}\left\langle\mathbf{x}, M\left(t, \omega_{0}, M_{0}\right) \mathbf{x}\right\rangle .
$$

Since $M\left(t, \omega_{0}, M_{0}\right)$ is positive semidefinite we deduce that $V^{\omega_{0}}(t, \mathbf{x}) \geq 0$. As in [15], a straightforward computation from the Riccati equation (4.11) satisfied by $M\left(t, \omega_{0}, M_{0}\right)$ shows that

$$
\begin{aligned}
\frac{d V^{\omega_{0}}}{d t}(t, \mathbf{x}(t))= & \mathcal{Q}_{\omega_{0}}(t, \mathbf{x}(t), \mathbf{u}(t)) \\
& -\frac{1}{2}\left\langle\mathbf{u}(t)-K^{\omega_{0}}(t) \mathbf{x}(t), R\left(\omega_{0} \cdot t\right)\left(\mathbf{u}(t)-K^{\omega_{0}}(t) \mathbf{x}(t)\right)\right\rangle,
\end{aligned}
$$

where $K^{\omega_{0}}(t)=R^{-1}\left(\omega_{0} \cdot t\right)\left(B^{T}\left(\omega_{0} \cdot t\right) M\left(t, \omega_{0}, M_{0}\right)-g^{T}\left(\omega_{0} \cdot t\right)\right)$ and $\mathbf{x}(t)$ satisfies (4.4). Therefore, since $R>0$,

$$
\int_{t_{1}}^{t_{2}} \mathcal{Q}_{\omega_{0}}(t, \mathbf{x}(t), \mathbf{u}(t)) d t \geq V^{\omega_{0}}\left(t_{1}, \mathbf{x}\left(t_{2}\right)\right)-V^{\omega_{0}}\left(t_{1}, \mathbf{x}\left(t_{1}\right)\right),
$$

that is, $V^{\omega_{0}}$ is a storage function for the control system (4.4) with supply rate $\mathcal{Q}_{\omega_{0}}$, which proves (i).

(ii) Let $K$ be the closure of the trajectory of $\left(\omega_{0}, M_{0}\right)$. Since $\omega_{0}$ has dense orbit, for each $\omega \in \Omega$ we can find $M_{\omega}$ such that $\left(\omega, M_{\omega}\right) \in K$. It is easy to deduce from the continuity of the Riccati flow (2.6) that $M\left(t, \omega, M_{\omega}\right)$ is defined and positive semidefinite for all $t \in \mathbb{R}$. Once this has been established, (ii) follows from (i).

Remark 4.16. A similar result for the dissipativity of the time-reversed control system (4.6) is obtained if the conditions

- $M_{0} \geq 0$ and $M\left(t, \omega_{0}, M_{0}\right)$ is globally defined and positive semidefinite for all $t \in \mathbb{R}$

in Proposition 4.15(i) are modified to read

- $M_{0} \leq 0$ and $M\left(t, \omega_{0}, M_{0}\right)$ is globally defined and negative semidefinite for all $t \in \mathbb{R}$.

Assume now that the family of control systems (4.4) is not uniformly null controllable and the family (4.8) admits exponential dichotomy. Applying Theorem 3.4 to one of the minimal subsets $\Omega^{*} \subset \Omega$ for which all the systems are abnormal, we deduce that $\widetilde{d}^{+}$or $\widetilde{d}^{-}$is strictly positive, that is, at least one of the associated Lagrange planes $l^{ \pm}(\omega)$ lies on the vertical Maslov cycle $\mathcal{C}$, defined in Subsection 2.3, for all $\omega \in \Omega^{*}$. The next result provides conditions for the dissipativity of the family 
of control systems (4.4) and the family of time-reversed control systems (4.6) when the other Lagrange plane lies in $\mathcal{D}=\mathcal{L}_{\mathbb{R}}-\mathcal{C}$.

Proposition 4.17. (i) Assume that the family (4.8) admits exponential dichotomy with $l^{-}(\omega) \in \mathcal{D}$ for all $\omega \in \Omega$. Represent $l^{-}(\omega) \equiv\left[\begin{array}{c}I_{n} \\ M^{-}(\omega)\end{array}\right] \in \mathcal{L}_{\mathbb{R}}$, and define

Then,

$$
V^{\omega}(t, \mathbf{x})=\frac{1}{2}\left\langle\mathbf{x}, M^{-}(\omega \cdot t) \mathbf{x}\right\rangle
$$

- if $M^{-}(\omega) \geq 0$ for all $\omega \in \Omega$, then the control system (4.4) is dissipative with supply rate $\mathcal{Q}_{\omega}$ and storage function $V^{\omega}$ for all $\omega \in \Omega$;

- if $M^{-}(\omega)>0$ for all $\omega \in \Omega$, then the control system (4.4) is strictly dissipative with supply rate $\mathcal{Q}_{\omega}$ and strong storage function $V^{\omega}$ for all $\omega \in \Omega$.

(ii) Assume that the family (4.8) admits exponential dichotomy with $l^{+}(\omega) \in \mathcal{D}$. Represent $l^{+}(\omega) \equiv\left[\begin{array}{c}I_{n} \\ M^{+}(\omega)\end{array}\right] \in \mathcal{L}_{\mathbb{R}}$ for each $\omega \in \Omega$, and define

Then,

$$
\widetilde{V}^{\omega}(t, \mathbf{x})=-\frac{1}{2}\left\langle\mathbf{x}, M^{+}(\omega \cdot t) \mathbf{x}\right\rangle \text {. }
$$

- if $M^{+}(\omega) \leq 0$ for all $\omega \in \Omega$, then the time-reversed control system (4.6) is dissipative with supply rate $\mathcal{Q}_{\omega}^{-}$and storage function $\widetilde{V}^{\omega}$ for all $\omega \in \Omega$;

- if $M^{+}(\omega)<0$ for all $\omega \in \Omega$, then time-reversed control system (4.6) is strictly dissipative with supply rate $\mathcal{Q}_{\omega}^{-}$and strong storage function $\widetilde{V}^{\omega}$ for all $\omega \in \Omega$.

Proof. Since $M\left(t, \omega, M^{-}(\omega)\right)=M^{-}(\omega \cdot t)$, the first assertion in (i) follows from Proposition 4.15(i). For the second one, if $M^{-}(\omega)>0$, define

$$
G^{\delta}(\omega)=G(\omega)-\delta I_{n}, \quad R^{\delta}(\omega)=R(\omega)-\delta I_{m}
$$

where $\delta>0$ is chosen so that $R^{\delta}>0$, and consider the family of systems

$$
\mathbf{z}^{\prime}=H^{\delta}(\omega \cdot t) \mathbf{z}
$$

for $\omega \in \Omega$, obtained by substituting $G$ and $R$ by $G^{\delta}$ and $R^{\delta}$ in (4.8). The robustness of the exponential dichotomy ensures that, for $\delta>0$ small enough, the family (4.13) admits exponential dichotomy over $\Omega$; that the corresponding Lagrange plane $l_{\delta}^{-}(\omega)$ belongs to $\mathcal{D}$ for all $\omega \in \Omega: l_{\delta}^{-}(\omega)=\left[\begin{array}{c}I_{n} \\ M_{\delta}^{-}(\omega)\end{array}\right]$; and that $M_{\delta}^{-}(\omega) \geq 0$. Consequently, the control system (4.4) is dissipative with supply rate $\mathcal{Q}_{\omega}^{\delta}$, where

$$
\mathcal{Q}_{\omega}^{\delta}(t, \mathbf{x}, \mathbf{u})=\mathcal{Q}_{\omega}(t, \mathbf{x}, \mathbf{u})-\delta\left(\|\mathbf{x}\|^{2}+\|\mathbf{u}\|^{2}\right),
$$

and hence the control system (4.4) is strictly dissipative with supply rate $\mathcal{Q}_{\omega}$, as stated. Finally, since $V^{\omega}(t, \mathbf{x})>0$ for all $\mathbf{x} \neq 0$ and $t \in \mathbb{R}$, the storage function $V^{\omega}$ is strong.

We omit the proof of (ii), which is analogous.

The following result is a version of Proposition 5.9 of [16] when both Lagrange planes associated to the exponential dichotomy lie in the Maslov cycle $\mathcal{C}$. Note that the existence of an ergodic measure with full topological support is assumed in order to keep control of the disconjugacy of the perturbed family of Hamiltonian systems in terms of the exponential dichotomy property. 
Theorem 4.18. Assume that there is a $\sigma$-ergodic measure $m_{0}$ on $\Omega$ with full topological support; that the family (4.8) admits an exponential dichotomy; and that its rotation number with respect to $m_{0}$ is $\alpha=0$.

Then there is a $\rho>0$, such that, for each $\varepsilon \in(0, \rho)$, the family

$$
\mathbf{z}^{\prime}=H_{\varepsilon}(\omega \cdot t) \mathbf{z}=\left[\begin{array}{cc}
H_{1}(\omega \cdot t) & H_{3}(\omega \cdot t)+\varepsilon I_{n} \\
H_{2}(\omega \cdot t) & -H_{1}^{T}(\omega \cdot t)
\end{array}\right] \mathbf{z}
$$

has exponential dichotomy over $\Omega$. Moreover, the Lagrange planes $l_{\varepsilon}^{ \pm}(\omega)$ lie in $\mathcal{D}$ and if we represent $l_{\varepsilon}^{ \pm}(\omega) \equiv\left[\begin{array}{c}I_{n} \\ M_{\varepsilon}^{ \pm}(\omega)\end{array}\right] \in \mathcal{L}_{\mathbb{R}}$ for each $\omega \in \Omega$, then we have

$$
M_{\varepsilon_{1}}^{+}(\omega) \leq M_{\varepsilon_{2}}^{+}(\omega)<M_{\varepsilon_{2}}^{-}(\omega) \leq M_{\varepsilon_{1}}^{-}(\omega)
$$

whenever $0<\varepsilon_{1}<\varepsilon_{2}<\rho$.

Proof. The robustness of the exponential dichotomy provides a $\rho>0$ such that the family (4.14) admits an exponential dichotomy for $\varepsilon \in(0, \rho)$. In addition, its rotation number $\alpha_{\varepsilon}$ with respect to $m_{0}$, which is a fixed constant on $[0, \rho]$ (see [10]), vanishes. Since $H_{3}(\omega \cdot t)+\varepsilon I_{n}$ is positive definite for each $\varepsilon>0$ (and hence the family $\mathbf{x}^{\prime}=H_{1}(\omega \cdot t) \mathbf{x}+\left(H_{3}(\omega \cdot t)+\varepsilon I_{n}\right) \mathbf{u}$ is uniformly null controllable), Theorem 2 of [7] ensures that each of the systems of the family (4.14) is weakly disconjugate, i.e., there is a $t_{0}(\omega)$ such that for every nonzero solution $\mathbf{z}(t)=\left[\begin{array}{l}\mathbf{z}_{1}(t) \\ \mathbf{z}_{2}(t)\end{array}\right]$ with $\mathbf{z}_{1}(0)=0$, there holds $\mathbf{z}_{1}(t) \neq 0$ for all $t>t_{0}(\omega)$. Moreover, since Supp $m_{0}=\Omega$ and consequently there is a dense semiorbit in $\Omega$, this $t_{0}(\omega)$ can be taken to be the same for all the systems; that is, the family (4.14) is uniformly weakly disconjugate, as is shown in Theorem 5.5 of [14].

Finally, Theorem 5.7 of [16] proves that $l_{\varepsilon}^{ \pm}(\omega)$ lie in $\mathcal{D}$ for each $\omega \in \Omega$, and the comparison result given in Proposition 5.5 together with Theorem 5.6 of [16] provide $M_{\varepsilon_{1}}^{+}(\omega) \leq M_{\varepsilon_{2}}^{+}(\omega)<M_{\varepsilon_{2}}^{-}(\omega) \leq M_{\varepsilon_{1}}^{-}(\omega)$ whenever $0<\varepsilon_{1}<\varepsilon_{2}<\rho$, as stated.

Notice that, since $H_{3}(\omega \cdot t)+\varepsilon I_{n}>0$, Remark 5.6 of [14] ensures that all the systems of the family (4.14) are in fact disconjugate, i.e., for every nonzero solution $\mathbf{z}(t)=\left[\begin{array}{l}\mathbf{z}_{1}(t) \\ \mathbf{z}_{2}(t)\end{array}\right]$, the vector $\mathbf{z}_{1}(t)$ vanishes at most once in $\mathbb{R}$.

The next result provides conditions for dissipativity of the family of control systems (4.4) and the family of time-reversed control systems (4.6) in terms of the perturbed family of Hamiltonian systems (4.14). This applies to the case in which the family (4.8) admits an exponential dichotomy but one or both of the associated Lagrange planes lie in the Maslov cycle $\mathcal{C}$ and Proposition 4.17 cannot be applied.

Theorem 4.19. With the same hypotheses and notation of Theorem 4.18:

(i) Define

$$
V_{\varepsilon}^{\omega}(t, \mathbf{x})=\frac{1}{2}\left\langle\mathbf{x}, M_{\varepsilon}^{-}(\omega \cdot t) \mathbf{x}\right\rangle
$$

for each $\varepsilon \in(0, \rho)$. Then,

- if there is an $\varepsilon \in(0, \rho)$ such that $M_{\varepsilon}^{-}(\omega) \geq 0$ for all $\omega \in \Omega$, then the control system (4.4) is dissipative with supply rate $\mathcal{Q}_{\omega}$ and storage function $V_{\varepsilon}^{\omega}$ for all $\omega \in \Omega$;

- if there is an $\varepsilon \in(0, \rho)$ such that $M_{\varepsilon}^{-}(\omega)>0$ for all $\omega \in \Omega$, then the control system (4.4) is strictly dissipative with supply rate $\mathcal{Q}_{\omega}$ and strong storage function $V_{\varepsilon}^{\omega}$ for all $\omega \in \Omega$. 
(ii) Define

$$
\widetilde{V}_{\varepsilon}^{\omega}(t, \mathbf{x})=-\frac{1}{2}\left\langle\mathbf{x}, M_{\varepsilon}^{+}(\omega \cdot t) \mathbf{x}\right\rangle
$$

for each $\varepsilon \in(0, \rho)$. Then,

- if there is an $\varepsilon \in(0, \rho)$ such that $M_{\varepsilon}^{+}(\omega) \leq 0$ for all $\omega \in \Omega$, then the time-reversed control system (4.6) is dissipative with supply rate $\mathcal{Q}_{\omega}^{-}$ and storage function $\widetilde{V}_{\varepsilon}^{\omega}$ for all $\omega \in \Omega$;

- if there is an $\varepsilon \in(0, \rho)$ such that $M_{\varepsilon}^{+}(\omega)<0$ for all $\omega \in \Omega$, then the time-reversed control system (4.6) is strictly dissipative with supply rate $\mathcal{Q}_{\omega}^{-}$and strong storage function $V_{\varepsilon}^{\omega}$ for all $\omega \in \Omega$.

Proof. (i) Assume that there is an $\varepsilon \in(0, \rho)$ such that $M_{\varepsilon}^{-}(\omega) \geq 0$ for all $\omega \in \Omega$. We claim that for each $t \geq 0$,

$$
\begin{aligned}
\frac{d}{d t} V_{\varepsilon}^{\omega}(t, \mathbf{x}(t)) \leq & \mathcal{Q}_{\omega}(t, \mathbf{x}(t), \mathbf{u}(t)) \\
& -\frac{1}{2}\left\langle\mathbf{u}(t)-K_{\varepsilon}(\omega \cdot t) \mathbf{x}(t), R(\omega \cdot t)\left(\mathbf{u}(t)-K_{\varepsilon}(\omega \cdot t) \mathbf{x}(t)\right)\right\rangle,
\end{aligned}
$$

where $K_{\varepsilon}(\omega)=R^{-1}(\omega)\left(B^{T}(\omega) M_{\varepsilon}^{-}(\omega)-g(\omega)^{T}\right)$ and $\mathbf{x}(t)$ satisfies (4.4).

Using the action of the flow on $\Omega$ and an appropriate time translation of the control, it is enough to check (4.15) at $t=0$. As before, we denote by $M\left(t, \omega, M_{\varepsilon}^{-}(\omega)\right)$ the solution of the Riccati equation associated to (4.8) (that is, $\varepsilon=0$ ) with initial data $M_{\varepsilon}^{-}(\omega)$. Since $M_{\varepsilon}^{-}(\omega \cdot t)$ is the solution of the Riccati equation associated to (4.14) with the same initial data, it is easy to check that

$$
\left.\frac{d}{d t} M_{\varepsilon}^{-}(\omega \cdot t)\right|_{t=0} \leq\left. M^{\prime}\left(t, \omega, M_{\varepsilon}^{-}(\omega)\right)\right|_{t=0},
$$

and hence, as in (4.12),

$$
\begin{aligned}
\left.\frac{d}{d t} V_{\varepsilon}^{\omega}(t, \mathbf{x}(t))\right|_{t=0} \leq & \left.\frac{1}{2} \frac{d}{d t}\left\langle\mathbf{x}(t), M\left(t, \omega, M_{\varepsilon}^{-}(\omega)\right) \mathbf{x}(t)\right\rangle\right|_{t=0}=\left.\mathcal{Q}_{\omega}(t, \mathbf{x}(t), \mathbf{u}(t))\right|_{t=0} \\
& -\left.\frac{1}{2}\left\langle\mathbf{u}(t)-K_{\varepsilon}^{\omega}(t) \mathbf{x}(t), R(\omega \cdot t)\left(\mathbf{u}(t)-K_{\varepsilon}^{\omega}(t) \mathbf{x}(t)\right)\right\rangle\right|_{t=0}
\end{aligned}
$$

where $K_{\varepsilon}^{\omega}(t)=R^{-1}(\omega \cdot t)\left(B^{T}(\omega \cdot t) M\left(t, \omega, M_{\varepsilon}^{-}(\omega)\right)-g^{T}(\omega \cdot t)\right)$. This proves the claim for $t=0$ because $K_{\varepsilon}^{\omega}(0)=K_{\varepsilon}(\omega)$. Integrating (4.15) in $\left[t_{1}, t_{2}\right]$ and having in mind that $R>0$ we obtain

$$
\int_{t_{1}}^{t_{2}} \mathcal{Q}_{\omega}(t, \mathbf{x}(t), \mathbf{u}(t)) d t \geq V_{\varepsilon}^{\omega}\left(t_{2}, \mathbf{x}\left(t_{2}\right)\right)-V_{\varepsilon}^{\omega}\left(t_{1}, \mathbf{x}\left(t_{1}\right)\right),
$$

that is, $V_{\varepsilon}^{\omega}$ is a storage function for the control system (4.4) with supply rate $\mathcal{Q}_{\omega}$ and the control system (4.4) is dissipative with supply rate $\mathcal{Q}_{\omega}$, as stated. Finally, as in the proof of Proposition 4.17(i), the storage function $V_{\varepsilon}^{\omega}$ is strong if $M_{\varepsilon}^{-}>0$.

We omit the proof of (ii) which is analogous to the proof of (i).

4.2. Examples. We next describe two examples showing the applicability of Theorem 4.19 to study the dissipativity of some nonautonomous systems without uniform null controllability.

Johnson and Núñez [15] construct an almost-periodic uniformly null controllable LQ family of dissipative systems for which the associated family of linear Hamiltonian system is uniformly weakly disconjugate, has positive upper Lyapunov exponent 
and does not have exponential dichotomy. We show that such a situation can also occur when the LQ family of control systems is not null controllable and its dissipativity can be analyzed by a modified version of Theorem 4.19.

Using a method due to Millionšcikov and Vinograd, they obtain a family of limit-periodic linear Hamiltonian systems

$$
\mathbf{z}^{\prime}=\left[\begin{array}{cc}
-1-\widetilde{g}(\omega \cdot t) & 1 \\
-1 & 1+\widetilde{g}(\omega \cdot t)
\end{array}\right] \mathbf{z}
$$

with Lyapunov exponent $\gamma>0$ and Sacker-Sell spectrum $[-\gamma, \gamma]$. The family (4.16) is disconjugate and the principal solutions determine the Lagrange planes $l_{0}^{+}(\omega) \equiv$ $\left[\begin{array}{c}1 \\ n^{+}(\omega)\end{array}\right], l_{0}^{-}(\omega) \equiv\left[\begin{array}{c}1 \\ n^{-}(\omega)\end{array}\right]$ associated to the negative and positive Lyapunov exponents respectively. Moreover,

$$
2-\sqrt{3} \leq n^{+}(\omega) \leq n^{-}(\omega) \leq 2+\sqrt{3}
$$

for each $\omega \in \Omega$. From Theorem 4.4 of [13] and Theorem 5.6 of [16] we deduce that for each $\lambda \in(-\infty, 0)$ the family of linear Hamiltonian systems

$$
\mathbf{z}^{\prime}=\left[\begin{array}{cc}
-1-\widetilde{g}(\omega \cdot t) & 1 \\
-1-\lambda & 1+\widetilde{g}(\omega \cdot t)
\end{array}\right] \mathbf{z}
$$

has exponential dichotomy with corresponding Lagrange planes $l_{\lambda}^{+}(\omega) \equiv\left[\begin{array}{c}1 \\ n^{+}(\omega, \lambda)\end{array}\right]$, $l_{\lambda}^{-}(\omega) \equiv\left[\begin{array}{c}1 \\ n^{-}(\omega, \lambda)\end{array}\right]$ satisfying

$$
n^{+}\left(\omega, \lambda_{2}\right) \leq n^{+}\left(\omega, \lambda_{1}\right)<n^{-}\left(\omega, \lambda_{1}\right) \leq n^{-}\left(\omega, \lambda_{2}\right)
$$

whenever $\lambda_{2}<\lambda_{1}<0$, and

$$
\lim _{\lambda \rightarrow 0^{-}} n^{ \pm}(\omega, \lambda)=n^{ \pm}(\omega)
$$

If we fix $\lambda<0$, the robustness of the exponential dichotomy ensures that for $\varepsilon>0$ small enough the family of linear Hamiltonian systems

$$
\mathbf{z}^{\prime}=\left[\begin{array}{cc}
-1-\widetilde{g}(\omega \cdot t) & 1+\varepsilon \\
-1-\lambda & 1+\widetilde{g}(\omega \cdot t)
\end{array}\right] \mathbf{z}
$$

also admits an exponential dichotomy with associated Lagrange planes $\left[\begin{array}{c}1 \\ n_{\varepsilon}^{ \pm}(\omega, \lambda)\end{array}\right]$, and from (4.18) and (4.19) we deduce that $0<n^{+}(\omega)<n^{-}(\omega, \lambda)$, and hence for $\varepsilon$ small enough $n_{\varepsilon}^{-}(\omega, \lambda)>0$.

Next we consider $R=I_{3}, \widetilde{G}(\omega)=\widetilde{g}^{2}(\omega)-1$, the diagonal matrices

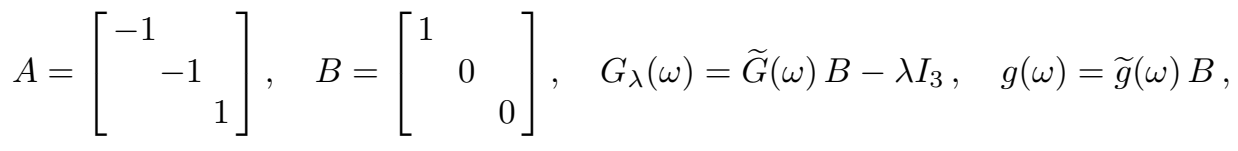

and the family of linear control systems

$$
\mathbf{x}^{\prime}=A \mathbf{x}+B \mathbf{u}
$$

with family of quadratic functionals

$$
\mathcal{Q}_{\omega}^{\lambda}(t, \mathbf{x}, \mathbf{u})=\frac{1}{2}\left(\left\langle\mathbf{x}, G_{\lambda}(\omega \cdot t) \mathbf{x}\right\rangle+2\langle\mathbf{x}, g(\omega \cdot t) \mathbf{u}\rangle+\langle\mathbf{u}, \mathbf{u}\rangle\right) .
$$

The associated family of linear Hamiltonian systems is

$$
\mathbf{z}^{\prime}=\left[\begin{array}{cc}
A-\widetilde{g}(\omega \cdot t) B & B \\
-B-\lambda I_{3} & -A+\widetilde{g}(\omega \cdot t) B
\end{array}\right] \mathbf{z} .
$$


Now we fix $\lambda<0$. Taking into account that this family uncouples into three two-dimensional families: the family (4.17) and the constant coefficient ones

$$
\mathbf{z}^{\prime}=\left[\begin{array}{ll}
-1 & 0 \\
-\lambda & 1
\end{array}\right] \mathbf{z} \quad \text { and } \quad \mathbf{z}^{\prime}=\left[\begin{array}{rr}
1 & 0 \\
-\lambda & -1
\end{array}\right] \mathbf{z},
$$

we deduce that it admits exponential dichotomy with Lagrange planes

$$
l^{+}(\omega, \lambda) \equiv\left[\begin{array}{ccc}
1 & 0 & 0 \\
0 & 1 & 0 \\
0 & 0 & 0 \\
n^{+}(\omega, \lambda) & 0 & 0 \\
0 & \lambda / 2 & 0 \\
0 & 0 & 1
\end{array}\right] \quad \text { and } \quad l^{-}(\omega, \lambda) \equiv\left[\begin{array}{ccc}
1 & 0 & 0 \\
0 & 0 & 0 \\
0 & 0 & 1 \\
n^{-}(\omega, \lambda) & 0 & 0 \\
0 & 1 & 0 \\
0 & 0 & -\lambda / 2
\end{array}\right]
$$

with indices $d^{+}(\omega)=d^{-}(\omega)=1$ for every $\omega \in \Omega$. Again, for $\varepsilon>0$ small enough the family of perturbed systems

$$
\mathbf{z}^{\prime}=\left[\begin{array}{cc}
A-\widetilde{g}(\omega \cdot t) B & B+\varepsilon I_{3} \\
-B-\lambda I_{3} & -A+\widetilde{g}(\omega \cdot t) B
\end{array}\right] \mathbf{z}
$$

also admits an exponential dichotomy with Lagrange planes $\left[\begin{array}{c}I_{3} \\ N_{\varepsilon}^{ \pm}(\omega, \lambda)\end{array}\right]$, where

$$
\begin{aligned}
& N_{\varepsilon}^{+}(\omega, \lambda)=\left[\begin{array}{ccc}
n_{\varepsilon}^{+}(\omega, \lambda) & 0 & 0 \\
0 & \frac{1-\sqrt{1-\varepsilon \lambda}}{\varepsilon} & 0 \\
0 & 0 & \frac{-1-\sqrt{1-\varepsilon \lambda}}{\varepsilon}
\end{array}\right] \text { and } \\
& N_{\varepsilon}^{-}(\omega, \lambda)=\left[\begin{array}{ccc}
n_{\varepsilon}^{-}(\omega, \lambda) & 0 & 0 \\
0 & \frac{1+\sqrt{1-\varepsilon \lambda}}{\varepsilon} & 0 \\
0 & 0 & \frac{-1+\sqrt{1-\varepsilon \lambda}}{\varepsilon}
\end{array}\right] .
\end{aligned}
$$

Hence $N_{\varepsilon}^{-}(\omega, \lambda)>0$ for $\varepsilon>0$ small enough, and Theorem 4.19 shows that the control system (4.20) is strictly dissipative with supply rate $\mathcal{Q}_{\omega}^{\lambda}$ given by (4.21).

As a second example, consider the family of perturbed linear Hamiltonian systems

$$
\mathbf{z}^{\prime}=\left[\begin{array}{cc}
A-\widetilde{g}(\omega \cdot t) B & B+\varepsilon\left(I_{3}-B\right) \\
-B-\lambda I_{3} & -A+\widetilde{g}(\omega \cdot t) B
\end{array}\right] \mathbf{z}
$$

which for $\lambda<0$ and each $\varepsilon>0$ admit an exponential dichotomy with Lagrange planes $\left[\begin{array}{c}I_{3} \\ \widetilde{N}_{\varepsilon}^{ \pm}(\omega, \lambda)\end{array}\right]$, where

$$
\begin{aligned}
& \widetilde{N}_{\varepsilon}^{+}(\omega, \lambda)=\left[\begin{array}{ccc}
n^{+}(\omega, \lambda) & 0 & 0 \\
0 & \frac{1-\sqrt{1-\varepsilon \lambda}}{\varepsilon} & 0 \\
0 & 0 & \frac{-1-\sqrt{1-\varepsilon \lambda}}{\varepsilon}
\end{array}\right], \\
& \widetilde{N}_{\varepsilon}^{-}(\omega, \lambda)=\left[\begin{array}{ccc}
n^{-}(\omega, \lambda) & 0 & 0 \\
0 & \frac{1+\sqrt{1-\varepsilon \lambda}}{\varepsilon} & 0 \\
0 & 0 & \frac{-1+\sqrt{1-\varepsilon \lambda}}{\varepsilon}
\end{array}\right],
\end{aligned}
$$

and hence, since $B+\varepsilon\left(I_{3}-B\right)>0$ implies that the systems are identically normal, the systems are disconjugate, as shown in Chapter 2.1 of Coppel [1]. As a consequence, as in the proof of Theorem 4.4 of [13] we check that for $\lambda=0$ and for each 
$\varepsilon>0$, the family $(4.22)$ is disconjugate with principal solutions $\widetilde{N}_{\varepsilon}^{ \pm}(\omega)$ given by

$$
\begin{aligned}
& \widetilde{N}_{\varepsilon}^{+}(\omega)=\lim _{\lambda \rightarrow 0^{-}} \widetilde{N}_{\varepsilon}^{+}(\omega, \lambda)=\left[\begin{array}{ccc}
n^{+}(\omega) & 0 & 0 \\
0 & 0 & 0 \\
0 & 0 & -2 / \varepsilon
\end{array}\right], \\
& \widetilde{N}_{\varepsilon}^{-}(\omega)=\lim _{\lambda \rightarrow 0^{-}} \widetilde{N}_{\varepsilon}^{-}(\omega, \lambda)=\left[\begin{array}{ccc}
n^{-}(\omega) & 0 & 0 \\
0 & 2 / \varepsilon & 0 \\
0 & 0 & 0
\end{array}\right] \geq 0,
\end{aligned}
$$

for each $\omega \in \Omega$. Finally, although the family (4.22) does not have an exponential dichotomy for $\lambda=0$, the same arguments used in the proof of Theorem 4.19 with

$$
V_{\varepsilon}^{\omega}(t, \mathbf{x})=\frac{1}{2}\left\langle\mathbf{x}, \widetilde{N}_{\varepsilon}^{-}(\omega \cdot t) \mathbf{x}\right\rangle
$$

show that, if $\lambda=0$, then the control system (4.20) is dissipative with supply rate $\mathcal{Q}_{\omega}^{0}$ given by (4.21).

\section{REFERENCES}

[1] W.A. Coppel, Disconjugacy, Lecture Notes in Mathematics 220, Springer-Verlag, Berlin, Heidelberg, New York, 1971.

[2] I.P. Cornfeld, S.V. Fomin, Ya.G. Sinai, Ergodic Theory, Springer-Verlag, Berlin, Heidelberg, New York, 1982.

[3] R. Ellis, Lectures on Topological Dynamics, Benjamin, New York, 1969.

[4] R. Fabbri, R. Johnson, S. Novo, C. Núñez, Some remarks concerning weakly disconjugate linear Hamiltonian systems, J. Math. Anal. Appl. 380 (2011), 853-864.

[5] R. Fabbri, R. Johnson, S. Novo, C. Núñez, On linear-quadratic dissipative control processes with time-varying coefficients, Discrete Contin. Dynam. Systems, Ser. A, 33 (1) (2013), 193-210.

[6] R. Fabbri, R. Johnson, C. Núñez, On the Yakubovich Frequency Theorem for linear nonautonomous control processes, Discrete Contin. Dynam. Systems, Ser. A 9 (3) (2003), $677-704$.

[7] R. Fabbri, R. Johnson, C. Núñez, Disconjugacy and the rotation number for linear, nonautonomous Hamiltonian systems, Ann. Mat. Pura App. 185 (2006), S3-S21.

[8] D.J. Hill, Dissipative nonlinear systems: basic properies and stability analysis, Proc. 31st IEEE Conference on Decision and Control, Vol. 4 (1992), 3259-3264.

[9] D.J. Hill, P.J. Moylan, Dissipative dynamical systems: Basic input-output and state properties, J. Franklin Inst., Vol. 309, No. 5 (1980), 327-357.

[10] R. Johnson, $m$-functions and Floquet exponents for linear differential systems, Ann. Mat. Pura Appl. 147 (1987), 211-248.

[11] R. Johnson, M. Nerurkar, Exponential dichotomy and rotation number for linear Hamiltonian systems, J. Differential Equations 108 (1994), 201-216.

[12] R. Johnson, M. Nerurkar, Controllability, stabilization, and the regulator problem for random differential systems, Mem. Amer. Math. Soc. 646, Amer. Math. Soc., Providence, 1998.

[13] R. Johnson, S. Novo, R. Obaya, An ergodic and topological approach to disconjugate linear Hamiltonian systems, Illinois J. Math. 45 (2001), 1045-1079.

[14] R. Johnson, S. Novo, C. Núñez, R. Obaya, Uniform weak disconjugacy and principal solutions for linear Hamiltonian systems, in: Recent Advances in Delay Differential and Difference Equations, Springer Proceedings in Mathematics \& Statistics 94, Springer International Publishing Switzerland 2014, 131-159.

[15] R. Johnson, C. Núñez, Remarks on linear-quadratic dissipative control systems, Discr. Cont. Dyn. Sys. B 20 (3) (2015), 889-914.

[16] R. Johnson, C. Núñez, R. Obaya, Dynamical methods for linear Hamiltonian systems with applications to control processes, J. Dynam. Differential Equations 25 (3) (2013), 679-713.

[17] T. Kato, Perturbation Theory for Linear Operators, Corrected Printing of the Second Edition, Springer-Verlag, Berlin, Heidelberg 1995. 
[18] W. Kratz, Quadratic Functionals in Variational Analysis and Control Theory, Mathematical Topics 6, Akademie Verlag, Berlin, 1995.

[19] W. Kratz, Definiteness of quadratic functionals, Analysis (Munich) 23 (2) (2003), 163-183.

[20] V.B. Lidskiǔ, Oscillation theorems for canonical systems of differential equations, Dokl. Akad. Nank. SSSR 102 (1955), 877-880. (English translation in: NASA Technical Translation, P14, 696.)

[21] Y. Matsushima, Differentiable Manifolds, Marcel Dekker, New York 1972.

[22] A.S. Mishchenko, V.E. Shatalov, B.Yu. Sternin, Lagrangian Manifolds and the Maslov Operator, Springer-Verlag, Berlin, Heidelberg, New York, 1990.

[23] S. Novo, C. Núñez, R. Obaya, Ergodic properties and rotation number for linear Hamiltonian systems, J. Differential Equations 148 (1998), 148-185.

[24] I.G. Polushin, Stability results for quasidissipative systems, Proc. 3rd Eur. Control Conference ECC'95, 1995, 681-686.

[25] W.T. Reid, Principal solutions of nonoscillatory linear differential systems, J. Math. Anal. Appl. 9 (1964), 397-423.

[26] W.T. Reid, Sturmian Theory for Ordinary Differential Equations, Applied Mathematical Sciences 31, Springer-Verlag, New York, 1980.

[27] R.J. Sacker, G.R. Sell, Dichotomies and invariant splittings for linear differential systems III, J. Differential Equations 22 (1976), 497-522.

[28] J.F. Selgrade, Isolated invariant sets for flows on vector bundles, Trans. Amer. Math. Soc. 203 (1975), 359-390

[29] P. Šepitka, R. Šimon Hilscher, Minimal principal solution at infinity for nonoscillatory linear Hamiltonian systems, J. Dynam. Differential Equations 26 (1) (2014), 57-91.

[30] P. Šepitka, R. Šimon Hilscher, Principal Solutions at Infinity of Given Ranks for Nonoscillatory Linear Hamiltonian Systems, J. Dynam. Differential Equations 27 (1) (2015), 137-175.

[31] A.V. Savkin, I.R. Petersen, Structured dissipativeness and absolute stability of nonlinear systems, Internat. J. Control 62 (1995), no. 2, 443-460.

[32] H.L. Trentelman, J.C. Willems, Storage functions for dissipative linear systems are quadratic state functions, Proc. 36th IEEE Conf. Decision and Control (1997), 42-49.

[33] M. Wahrheit, Eigenvalue problems and oscillation of linear Hamiltonian systems, International J. Difference Equ. 2 (2) (2007), 221-244.

[34] J.C. Willems, Dissipative dynamical systems. Part I: General theory. Part II: Linear systems with quadratic supply rates, Arch. Rational Mech. Anal. 45 (1972), 321-393.

[35] V.A. Yakubovich, Oscillatory properties of the solutions of canonical equations, Amer. Math. Soc. Transl. Ser. 242 (1964), 247-288.

[36] V.A. Yakubovich, A.L. Fradkov, D.J. Hill, A.V. Proskurnikov, Dissipativity of T-periodic linear systems, IEEE Trans. Automat. Control 52 (2007), no. 6, 1039-1047.

[37] V.A. Yakubovich, V.M. Starzhinskii, Linear Differential Equations with Periodic Coefficients, John Wiley \& Sons, Inc., New York, 1975.

(Russell Johnson) Dipartimento di Matematica e Informatica, Università di Firenze, Via di Santa Marta 3, 50139 Firenze, Italy.

(Sylvia Novo, Carmen Núñez, Rafael Obaya) Departamento de Matemática Aplicada, Universidad de Valladolid, Paseo del Cauce 59, 47011 Valladolid, Spain.

E-mail address, Russell Johnson: russellallan.johnson@unifi.it

E-mail address, Sylvia Novo: sylnov@wmatem.eis.uva.es

E-mail address, Carmen Núñez: carnun@wmatem.eis.uva.es

E-mail address, Rafael Obaya: rafoba@wmatem.eis.uva.es 